\title{
Emission from the circumgalactic medium: from cosmological zoom-in simulations to multiwavelength observables
}

\author{
R. Augustin,,${ }^{1,2 \star}$ S. Quiret, ${ }^{1}$ B. Milliard,,${ }^{1}$ C. Péroux,,${ }^{1,2}$ D. Vibert, ${ }^{1}$ J. Blaizot, ${ }^{3}$ \\ Y. Rasera, ${ }^{4}$ R. Teyssier ${ }^{\oplus},{ }^{5}$ S. Frank, ${ }^{6}$ J.-M. Deharveng, ${ }^{1}$ V. Picouet, ${ }^{1}$ D. C. Martin, ${ }^{7}$ \\ E. T. Hamden,${ }^{7,8}$ N. Thatte, ${ }^{9}$ M. Pereira Santaella ${ }^{\oplus},{ }^{9}$ L. Routledge ${ }^{9}$ and \\ S. Zieleniewski ${ }^{9}, 10$ \\ ${ }^{1}$ CNRS, LAM (Laboratoire d'Astrophysique de Marseille), Aix Marseille Université, UMR 7326, F-13388, Marseille, France \\ ${ }^{2}$ European Southern Observatory, Karl-Schwarzschildstrasse 2, D-85748 Garching bei München, Germany \\ ${ }^{3}$ Ens de Lyon, CNRS, Centre de Recherche Astrophysique de Lyon UMR5574, Univ Lyon, Univ Lyon1, F-69230 Saint-Genis-Laval, France \\ ${ }^{4}$ LUTH, Observatoire de Paris, PSL Research University, CNRS, Université Paris Diderot, Sorbonne Paris Cité, 5 Place Jules Janssen, F-92195 Meudon, \\ France \\ ${ }^{5}$ Institute for Computational Science, University of Zürich, Winterthurerstrasse 190, CH-8057 Zürich, Switzerland \\ ${ }^{6}$ Department of Astronomy, The Ohio State University, Columbus, OH 43210, USA \\ ${ }^{7}$ California Institute of Technology, MC 405-47, 1200 East California Boulevard, Pasadena, CA 91125, USA \\ ${ }^{8}$ Steward Observatory, University of Arizona, 933 N Cherry Ave, Tucson, AZ 85721, USA \\ ${ }^{9}$ Department of Physics, University of Oxford, Denys Wilkinson Building, Keble Road, Oxford OX1 3RH, UK \\ ${ }^{10}$ King's College School, Southside, Wimbledon Common, London SW19 4TT, UK
}

Accepted 2019 August 8. Received 2019 August 8; in original form 2018 November 14

\begin{abstract}
We simulate the flux emitted from galaxy haloes in order to quantify the brightness of the circumgalactic medium (CGM). We use dedicated zoom-in cosmological simulations with the hydrodynamical adaptive mesh refinement code RAMSES, which are evolved down to $z=0$ and reach a maximum spatial resolution of $380 \mathrm{~h}^{-1} \mathrm{pc}$ and a gas mass resolution up to $1.8 \times 10^{5} h^{-1} \mathrm{M}_{\odot}$ in the densest regions. We compute the expected emission from the gas in the CGM using CLOUDY emissivity models for different lines (e.g. Ly $\alpha$, C IV, O VI, C VI, O VIII) considering UV background fluorescence, gravitational cooling and continuum emission. In the case of $\mathrm{Ly} \alpha$, we additionally consider the scattering of continuum photons. We compare our predictions to current observations and find them to be in good agreement at any redshift after adjusting the Ly $\alpha$ escape fraction. We combine our mock observations with instrument models for Faint Intergalactic Redshifted Emission Balloon-2 (FIREBall-2; UV balloon spectrograph) and HARMONI (visible and NIR IFU on the ELT) to predict CGM observations with either instrument and optimize target selections and observing strategies. Our results show that Ly $\alpha$ emission from the CGM at a redshift of 0.7 will be observable with FIREBall-2 for bright galaxies (NUV $18 \mathrm{mag}$ ), while metal lines like O VI and C IV will remain challenging to detect. HARMONI is found to be well suited to study the CGM at different redshifts with various tracers.
\end{abstract}

Key words: galaxies: evolution-galaxies: formation-intergalactic medium.

\section{INTRODUCTION}

Understanding the complex mechanisms regulating galaxy formation is one of the main questions today in cosmology and astrophysics. The question of how galaxies gather gas to sustain star formation is of particular interest, as it could shed light on the

^E-mail: ramona.augustin@lam.fr fact that the star formation rate (SFR) has been declining from $z \sim 2$ while diffusely distributed hydrogen still is the dominant component for the total baryonic mass budget (as compared to hydrogen in stars, Madau \& Dickinson 2014). Numerical simulations bring valuable insight into accretion mechanisms that replenish the gas reservoir of star formation. The two main mechanisms are cold accretion from dense flows of cold gas, and the hot accretion of more diffuse gas from the halo. Due to the current scarcity of direct observations, these are vividly debated (e.g. Kereš et al. 2005; Dekel et al. 
2009; Bournaud et al. 2011; Fox \& Davé 2017). Simultaneously, powerful gas outflows provide negative feedback on star formation. These outflows have been observed with various techniques and instruments, but their numerical implementation remains challenging (Pettini et al. 2001; Steidel et al. 2010; Vogelsberger et al. 2014).

The circumgalactic medium (CGM) of galaxies, at the interface between galaxies and the intergalactic medium (IGM), is loosely defined as the region within $\sim 300 \mathrm{kpc}$ (Steidel et al. 2010; Shull 2014; Tumlinson, Peeples \& Werk 2017) where these outflowing and accreting mechanisms are interacting. Studying the CGM will provide key constraints on the question of galaxy formation and evolution. Absorption spectroscopy has already shed light on the distribution and the chemical composition of the CGM gas, but only on a statistical point of view, given that only one line of sight per galaxy can be probed due to the scarcity of background quasars in the vicinity of galaxies (Noterdaeme et al. 2012; Pieri et al. 2014; Quiret et al. 2016; Rahmani et al. 2016; Krogager et al. 2017; Augustin et al. 2018). Hummels, Smith \& Silvia (2017) have implemented a technique to create mock absorption spectra from cosmological simulations in order to understand the gas we see in absorption, yet mapping the emission of the CGM is the natural next step to fully understanding the CGM. Its low surface brightness makes direct observation challenging, but there has been tremendous progress over the last years in order to find faint emission around galaxies. At high redshifts, large ground-based telescopes such as the Very Large Telescope (VLT), Subaru, or Keck offer the first hints of Ly $\alpha$ emission CGM mapping, achieved through the stacking of a large number of systems (Steidel et al. 2011; Momose et al. 2014), long exposures (Rauch et al. 2008; Wisotzki et al. 2016, 2018), or by selecting objects whose Ly $\alpha$ luminosity is boosted by the presence of a bright quasar nearby (Cantalupo et al. 2014; Martin et al. 2014; Borisova et al. 2016; Arrigoni Battaia et al. 2015, 2016, 2018, 2019). Gronke \& Bird (2017) have shown that indeed the findings so far agree with the Ly $\alpha$ profiles found from simulations and extended low surface brightness gas around galaxies. Using narrow-band imaging with HST, Hayes et al. (2016) have discovered also extended O VI emission around a $z=0.2$ galaxy and thereby created one of the first metal line maps of the CGM.

Understanding the different processes responsible for diffuse emission in this region is of great interest. An accurate modelling of these processes would require spatial resolution of a few parsecs and accounting for dust effects and radiative transfer, which is only currently achievable for single galaxy simulations (e.g. Rosdahl \& Blaizot 2012; Oppenheimer et al. 2016). At the same time, the study of co-evolution between galaxies and the IGM has to be conducted on much larger scales. Moreover, modelling the emission from this medium requires chemical-photoionization calculations, that are simply too heavy to be produced on the fly. Post-processing radiative transfer analysis is so far the best tool to estimate a realistic level of emission. Bertone \& Schaye (2012) have used the hydrodynamic cosmological simulation OWLS (Schaye et al. 2010) to analyse the strength of UV lines in the CGM and predicted the brightest emission line to be from H I Ly $\alpha(1216 \AA)$ and the strongest metal line to be C III (977 ̊). Silva, Kooistra \& Zaroubi (2016) have analytically studied the emission of $\operatorname{Ly} \alpha$ at $z<3$ in filaments and their detectability. They found that future spacebased experiments with a sensitivity of $3.7 \times 10^{-9} \mathrm{erg} \mathrm{s}^{-1} \mathrm{~cm}^{-2} \mathrm{sr}^{-1}$ $\left(\sim 37 \mathrm{mag} \mathrm{arcsec}^{-2}\right)$ will be able to detect hydrogen and helium in intergalactic filaments. Lokhorst et al. (2019) have used EAGLE simulations with a CLOUDY emission model to predict the fluxes from the CGM and IGM and investigated the detectability of faint $\mathrm{H} \alpha$ emission at low redshifts. They found that the Dragonfly Telephoto Array, ${ }^{1}$ equipped with suitable narrow-band filters, would be able to directly map the cosmic web.

Very recently, a number of cosmological simulations have highlighted the importance of increased resolution in the CGM in the context of making predictions for observations. Works by Hummels et al. (2018) and Suresh et al. (2019) have investigated the importance of highly resolved CGM in simulations in order to reproduce the observed cool gas column densities. Similarly, van de Voort et al. (2019) have found that the covering fraction of cool gas as traced by H I absorption increases dramatically with increasing spatial resolution. Corlies et al. (2018) and Peeples et al. (2019) have presented new Enzo AMR simulations with forced refinement in the CGM and made predictions for both absorption line studies as well as emission line maps as seen with current IFUs, confirming the resolution effect on CGM studies.

Here, we present a new simulation run of RAMSES (Teyssier 2002) over a box of 100 comoving $\mathrm{Mpc} h^{-1}$ with a zoom-in over a region of 13.92 comoving $\mathrm{Mpc} h^{-1}$ and our post-processing of snapshots to obtain flux maps and 3D data cubes of individual galaxies and their CGM. These are used to predict the expected flux of different lines to enable comparison with observations. We then use those 3D data cubes to create mock observations of the CGM with two different instruments: Faint Intergalactic Redshifted Emission Balloon-2 (FIREBall-2) and HARMONI.

This work is structured as follows: In Section 2, we present our cosmological zoom-in simulations, in Section 3 the photoionization model we are using, and in Section 4 the comparison to observations. In Section 5, we will use the simulated haloes to prepare FIREBall2 target selection and data analysis. We assess the compatibility of ELT/HARMONI for CGM observations in Section 6. Our conclusions are given in Section 7. We assume a flat $\Lambda \mathrm{CDM}$ universe with $\Omega_{\Lambda}=0.742, \Omega_{\mathrm{m}}=0.258, \Omega_{\mathrm{b}}=0.045, \mathrm{H} 0=71.9$, $\sigma_{8}=0.798, n_{\mathrm{s}}=0.963$.

\section{COSMOLOGICAL ZOOM-IN SIMULATIONS}

We use cosmological simulations that were produced with the RAMSES (Teyssier 2002) grid-based hydrodynamical solver with adaptive mesh refinement (AMR) using $\sim 1.3$ million CPU hours. The basis of this work is presented in Frank et al. (2012). They have used cosmological simulations and CLOUDY modelling to predict the line fluxes of three UV lines (Ly $\alpha$ at $1216 \AA$, O VI at 1032/1038 $\AA$ and C IV at $1548 / 1551 \AA$ ). They found that the CGM is expected to have high enough emission (e.g. $\log \left(L_{\mathrm{Ly} \alpha}\right) \sim 40.9-41.8 \mathrm{erg} \mathrm{s}^{-1}$ ) to be detectable with upcoming instruments but were less optimistic for filament detections from the IGM.

We aim to follow their approach in predicting CGM luminosities. However, many physical processes in gas clumps in and around galaxies are taking place on scales that are lower than what can be resolved in the simulations. With this in mind, we picked the most massive halo from the Frank et al. (2012) simulations (which is also the most luminous in Ly $\alpha$ in their simulation) and performed a zoom-in on the region around this halo. The halo was selected because of its high mass, which results in high-density gas cells. Indeed, in the AMR framework, the densest regions have the highest spatial resolution. This high spatial resolution allows us to distinguish between CGM and ISM and provides the basis for a

\footnotetext{
${ }^{1}$ http://www.dragonflytelescope.org/
} 
Table 1. Initial parameters for the zoom simulation. The parameters are analogous to Teyssier et al. (2013) and adjusted to the resolution in our zoom-in simulation.

\begin{tabular}{lcc}
\hline Parameter & Description & Value \\
\hline$\epsilon_{*}$ & Star formation efficiency & 0.01 \\
$n_{*}$ & Star formation density threshold in $\mathrm{H} \mathrm{cc}^{-1}$ & 3 \\
$T_{*}$ & ISM polytropic temperature in K/ $\mu$ & 3000 \\
$\eta_{\mathrm{sn}}$ & Supernova mass fraction & 0.2 \\
Yield & Supernova metal yield & 0.1 \\
mass $_{\mathrm{GMC}}$ & Stochastic exploding GMC mass in solar mass & $2 \times 10^{6}$ \\
$z_{\mathrm{reion}}$ & Reionization redshift & 20 \\
$\Omega_{\mathrm{m}}$ & Matter density & 0.26 \\
$\Omega_{\mathrm{l}}$ & Vacuum density & 0.74 \\
$\Omega_{\mathrm{b}}$ & Baryonic matter density & 0.045 \\
$\Omega_{\mathrm{k}}$ & Spatial curvature density & 0 \\
$H_{0}$ & Hubble parameter in km s & -1 \\
$\sigma_{8}$ & Amplitude of the (linear) power spectrum on the scale of $8 h^{-1} \mathrm{Mpc}$ & 71.9 \\
$n_{\mathrm{s}}$ & Primordial spectral index of scalar fluctuations & 0.798 \\
\hline
\end{tabular}

Table 2. Comparison of our zoom simulation with the low-resolution simulations of Frank et al. (2012).

\begin{tabular}{lcc}
\hline $\begin{array}{l}\text { Frank et al. (2012) } \\
\text { 'Low-resolution' simulation }\end{array}$ & Parameter & $\begin{array}{c}\text { Zoom-in (this work) } \\
\text { 'High-resolution' simulation }\end{array}$ \\
\hline $1.53 \mathrm{kpc} h^{-1}$ & Maximum spatial resolution & $0.38 \mathrm{kpc} h^{-1}$ \\
$\sim 4.42 \times 10^{8} \mathrm{M}_{\odot} h^{-1}$ & Maximum mass resolution for dark matter & $\sim 8.7 \times 10^{5} \mathrm{M}_{\odot} h^{-1}$ \\
$\sim 134 \times 10^{6}$ & Number of dark matter particles & $\sim 205 \times 10^{6}$ \\
$512^{3}$ & Number of initial gas cells & $128^{3}$ \\
100 comoving Mpc $h^{-1}$ & Box length & 13.92 comoving Mpc $h^{-1}$ \\
7 & Maximum level of refinement & 18 \\
\hline
\end{tabular}

detailed CGM study. The high mass of the halo could introduce a caveat towards low redshift $(z \lesssim 1)$ where this halo might not be representative of the average population. It corresponds to a massive group of galaxies rather than an isolated galaxy. However, at low redshifts, galaxies typically exist in groups and clusters, and thus the simulation will probe the CGM in realistic environments. We use the MUlti-Scale Initial Conditions code (Hahn, Abel \& Kaehler 2013) to zoom on a large cubic region with a box length of $13.92 \mathrm{Mpch}^{-1}$. The simulation was performed using nonthermal supernova (SN) feedback (Teyssier et al. 2013) and 'onthe-fly' self-shielding. The latter disables the ionizing background for cells with a neutral hydrogen density $n_{\mathrm{HI}}>0.01 \mathrm{atcc}^{-1}$. This reproduces the self-shielding of gas cells in dense regions from ionizing background radiation and gives a good prediction of the temperature in the absence of radiative transfer. The threshold value is based on radiative transfer studies that have derived an estimate on the density at which the fraction of neutral hydrogen becomes dominant (Faucher-Giguère et al. 2010; Rosdahl \& Blaizot 2012). The maximum refinement level is set to 18 , giving a spatial resolution in the densest region of the simulation of about $380 \mathrm{~h}^{-1}$ comoving parsecs and a typical resolution of 1-2 comoving kpc $h^{-1}$ in the CGM regions. A list of parameters describing the initial conditions (adapted from Teyssier et al. (2013) to the resolution in our simulations) of our simulations is given in Table 1 and a comparison with the previous low-resolution simulation is given in Table 2). The final number of particles is $\sim 205$ million for Dark Matter, 51 million for stars and 592 million gas cells, and at $z=0$, the central zoomed halo mass is about $3 \times 10^{13} h^{-1} \mathrm{M}_{\odot}$, with $\sim 30$ million particles. This makes it one of the largest $\mathrm{DM}+$ hydro+SF zoom simulations to date. Our analysis follows that of Frank et al. (2012) and Bertone \& Schaye (2012) with an increased resolution enabling us to probe colder and denser gas.
Fig. 1 shows six snapshots of the zoom-in region of the bright halo at different redshifts $(z=2.33,1.5,1.0,0.67,0.25,0.0$, respectively). We can see the progressive formation of the most massive halo from $z=1.0$. The web-like structure of the IGM clearly emerges in each of these snapshots, where we see faint filaments connecting overdense regions. We also witness the presence of isolated haloes within each filament. The properties of the most massive halo at different redshifts are gathered in Table 3. In this table, we only go down to a redshift of 0.25 because the processing of the haloes and calculation of those values for the $z=0$ snapshot take comparatively long and the data are not used in any later analysis.

Fig. 2 shows the density temperature diagram for the zoomed halo. The colours indicate the mass fraction of each bin in the $2 \mathrm{D}$ histogram. We find most of the gas to be in the IGM (low-density, high-temperature) region, where also most of the gas cells lie. There is a second, smaller peak of gas cells around the so-called gutter around $T \sim 10^{4} \mathrm{~K}$, where the cooling processes are in equilibrium with heating from external sources, which also holds a significant amount of gas mass. Most of the rest of the mass is in the ISM $(n>3$ at $\mathrm{cc}^{-1}$ ), although due to the resolution limit, these are concentrated in very few cells.

Fig. 3 shows the same diagram in red (but with the delayedcooling cells already taken away, see Section 3.1.3) and the same halo in the low-resolution (blue points) simulation. As expected, the high-resolution simulation extends to a larger parameter space, due to the higher number of cells in total in the halo. Two striking differences between these two simulations lie at the high density and low-temperature end of Fig. 3. First, as the resolution increases, gas with higher densities can be sampled near the centre of the gravitational well. This gas represents the high-density regions in the ISM. Consequently, the density threshold for star formation 

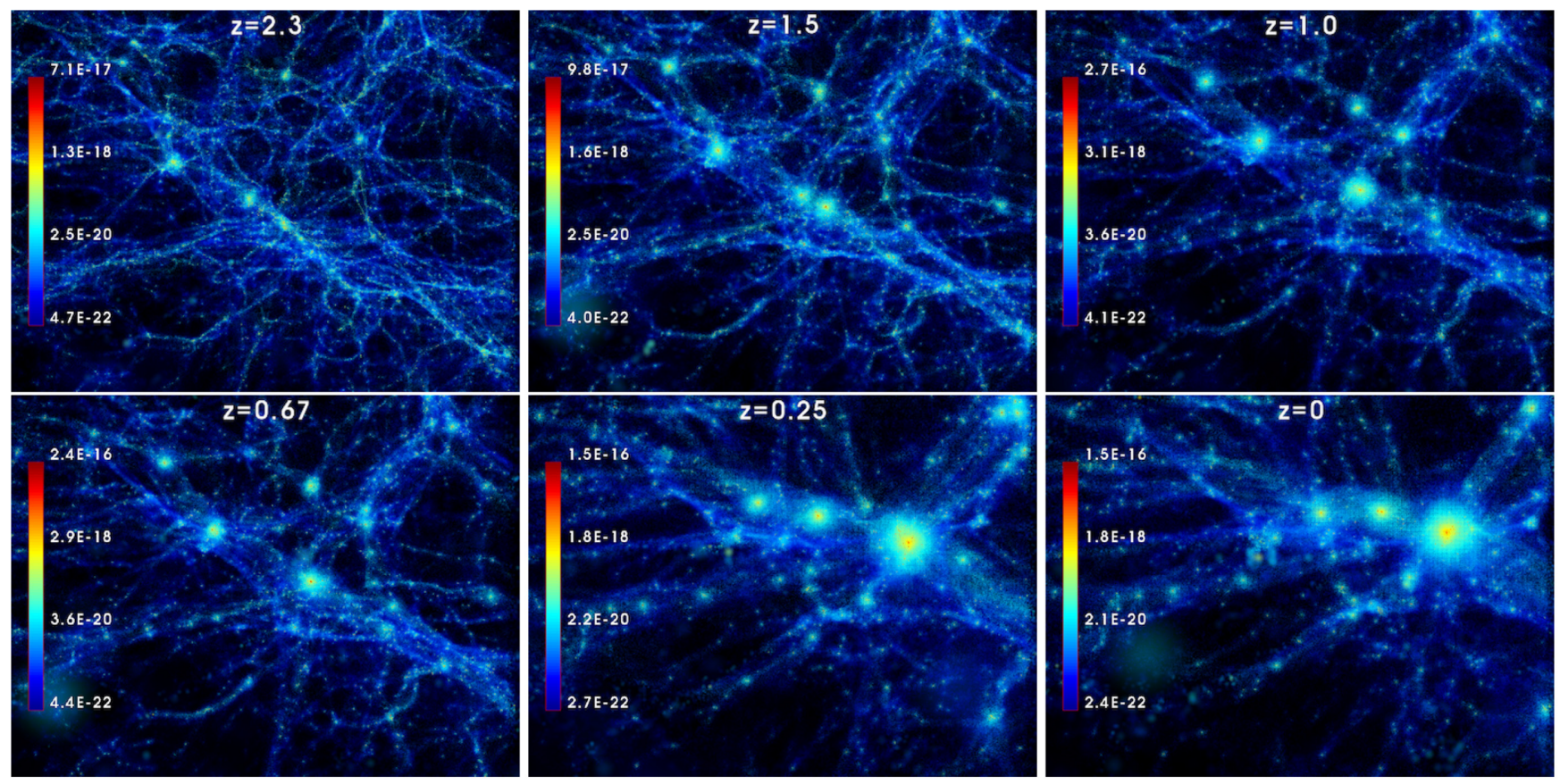

Figure 1. Evolution of the gas distribution in the high-resolution simulation for six different redshifts: $z=2.3,1.5,1.0,0.67,0.25,0$ (from top left to bottom right). Each panel corresponds to the 'zoomed' region of the high-resolution simulation, which is about 13.5 comoving Mpc $h^{-1}$. The filamentary structure of the IGM, and the formation of the massive haloes are apparent. The colour bar indicates the gas density in units of $\mathrm{kg} \mathrm{m}^{-3}$.

Table 3. Most massive halo characteristics for different redshifts. We give the redshift in the first column and the corresponding age of the Universe in the second. Columns 3-5 give the dark matter, stellar, and gas mass within the virial radius, which is given in physical as well as comoving units in columns 6 and 7 . Column 8 provides the star formation rate.

\begin{tabular}{lccccccc}
\hline$z$ & $\begin{array}{c}t_{\text {Universe }} \\
(\mathrm{Gyr})\end{array}$ & $\begin{array}{c}M_{\mathrm{DM}} \\
\left(10^{12} \mathrm{M}_{\odot}\right)\end{array}$ & $\begin{array}{c}M_{\star} \\
\left(10^{12} \mathrm{M}_{\odot}\right)\end{array}$ & $\begin{array}{c}M_{\mathrm{gas}} \\
\left(10^{12} \mathrm{M}_{\odot}\right)\end{array}$ & $\begin{array}{c}R_{\mathrm{vir}} \\
(\mathrm{pkpc})\end{array}$ & $\begin{array}{c}R_{\mathrm{vir}} \\
(\mathrm{ckpc})\end{array}$ & $\begin{array}{c}\mathrm{SFR} \\
\left(\mathrm{M}_{\odot} \mathrm{yr}^{-1}\right)\end{array}$ \\
\hline 9.0 & $\sim 0.6$ & 0.054 & 0.00015 & 0.005 & 9 & 94 & 2 \\
4.0 & $\sim 1.6$ & 0.6 & 0.013 & 0.09 & 55 & 274 & 95 \\
2.3 & $\sim 2.9$ & 2.4 & 0.122 & 0.26 & 128 & 423 & 351 \\
1.0 & $\sim 5.9$ & 9.6 & 0.852 & 1.36 & 327 & 655 & 622 \\
0.67 & $\sim 7.5$ & 13.6 & 1.11 & 1.61 & 391 & 652 & 246 \\
0.25 & $\sim 10.8$ & 28.3 & 3.37 & 4.0 & 679 & 848 & 333 \\
\hline
\end{tabular}

has been increased from $n_{\mathrm{H}}=0.1 \mathrm{atcc}^{-1}$ (Frank et al. 2012) to $n_{\mathrm{H}}=3$ at $\mathrm{cc}^{-1}$ at a star formation efficiency of 1 per cent. The cells following a polytropic floor in both simulations are an artefact from the simulation code to artificially stabilize the gas versus the Jeans criterion at the resolution limit.

The self-shielding of the gas leads to further gas cooling in the high-resolution simulation. The coupling of this 'on-the-fly' self-shielding option with the effect of the significantly higher resolution results in the emergence of dense and cool gas phase, for which gas cells reach temperatures below $10^{4} \mathrm{~K}$, with densities higher than $0.1 \mathrm{at} / \mathrm{cc}$. Such low temperatures could not be reached in the previous simulation set (Frank et al. 2012). These cells are clearly identified in the visual inspection of the simulation through the presence of discs. Examples are shown in Fig. A1 in the appendix. Here, the simulation reaches its limits as the spatial resolution in the high-density zones is of $381 \mathrm{pc} h^{-1}$ (comoving). Since we are not trying to resolve the ISM but are focused on the CGM, we conclude we reached the necessary limit in resolution where we can distinguish between galactic discs and the CGM.
Another addition to the zoom simulation is the implementation of non-thermal supernova (SN) feedback from Teyssier et al. (2013). In hydrodynamical cosmological simulations there are typically two ways to simulate the feedback from supernovae or AGN activity: the momentum-driven feedback and the energy-driven feedback (Costa, Sijacki \& Haehnelt 2014). The former injects pressure to the neighboring gas cells of a star particle undergoing supernova, acting a bit like a 'velocity kick', while the latter directly injects thermal energy and pushes the gas via adiabatic expansion of the hot shocked wind bubble. Costa et al. (2014) have shown that the momentum-driven solution is much less efficient in cosmological simulations than in isolated halo simulations, whereas the energy-driven solution is proven to be efficient in driving outflows also in large-scale (and therefore low-resolution) cosmological simulations. Therefore, we choose the energy-driven solution in our work. However, a major drawback of the energy-driven solution is that the injected energy is instantly radiated away by strong cooling, which appears to be a numerical effect of the simulation (Ceverino \& Klypin 2009). While other mechanisms, such as cosmic rays or magnetic fields, with longer dissipative time-scales are thought to sustain the pressure 


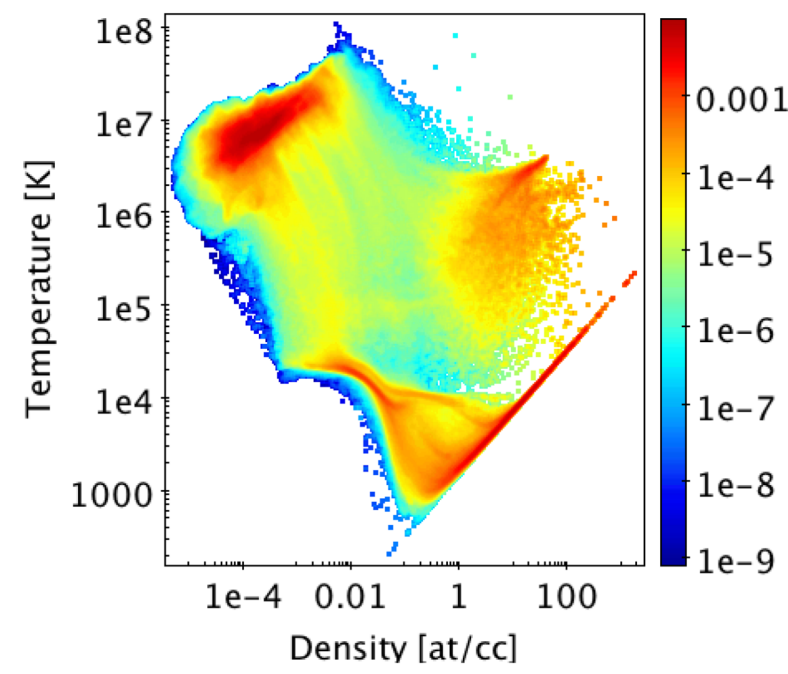

Figure 2. 2D histogram of the density-temperature phase diagram of the selected most massive halo of our zoom simulation at $z=0.67$. The density is given as total hydrogen density in at $\mathrm{cc}^{-1}$. The colour indicates the mass fraction of each bin (density bin width: $0.046 \mathrm{dex}$, temperature bin width: $0.054 \mathrm{dex}$ ). We find most of the gas in the IGM region (high temperatures, low densities).

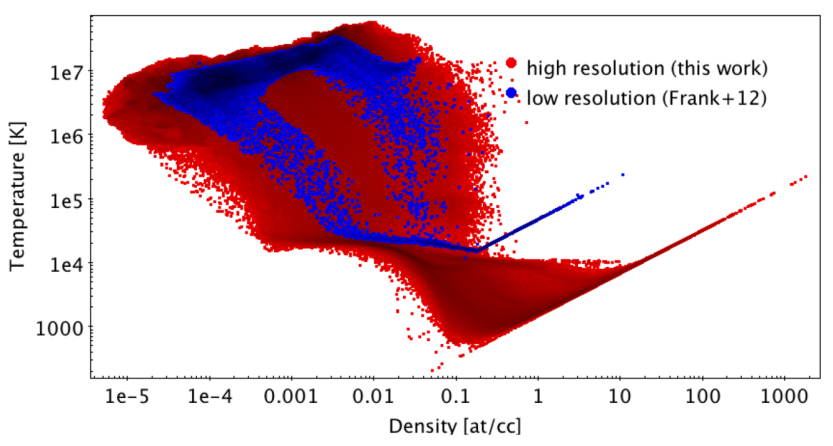

Figure 3. Density-temperature phase diagram of selected halo for lowresolution (up to $1.53 \mathrm{kpc} h^{-1}$, blue) and high-resolution simulations $\left(0.38 \mathrm{kpc} h^{-1}\right.$, red). The density is given as total hydrogen density in at $\mathrm{cc}^{-1}$. In our high-resolution simulation we can now reach lower temperatures and higher densities compared to the low-resolution simulations from Frank et al. (2012).

of the blast from this instant cooling (Cox 2005; Salem, Bryan \& Corlies 2016; Hopkins et al. 2019), we choose to momentarily stop the cooling of the gas after the energy injection. This feature, called 'delayed cooling', has been used in other works (Stinson et al. 2006; Governato et al. 2010; Agertz, Teyssier \& Moore 2011), and results in a temporary overestimate of the temperature of the affected cells. Those cells that are affected by the delayed cooling would, due to their artificially high temperature, cause a very strong line emission that is unrealistic. At redshift 0.7 these cells make up only around 0.5 per cent of all cells in the most massive halo. Therefore, in order to do an analysis on the line emission of gas around galaxies, we artificially remove these cells before post-processing the simulation snapshots.

\section{FLUX EMISSION PREDICTION}

The objective of this work is to put together a realistic model for faint diffuse emission from the CGM in order to prepare observations of the CGM with upcoming instruments. The model is set up such that we can make mock observations for any emission line from the CGM, e.g. typical UV lines such as Ly $\alpha$ at $1216 \AA$, O VI at $1032 / 1038 \AA$ or C IV at $1548 / 1551 \AA$, optical lines such as $\mathrm{H} \alpha$ at $6563 \AA$ or X-ray lines, such as O VIII at $19.0 \AA, \mathrm{C}$ VI at $33.7 \AA$ or NeIX at $13.4 \AA$. While we create a general model for any emission line, later in the analysis we will focus mainly on the UV line Ly $\alpha$ for comparison with observations and preparation for FIREBall-2. For the predictions for HARMONI we will mainly consider (redshifted) UV and optical lines. X-ray lines will be discussed in a subsequent publication. It is beyond the scope of the present analysis to propose specific improvements of the complex emission mechanisms from the CGM. Nevertheless, the high resolution reached on such largescale simulations brings innovative insight into CGM gas-phase emission line physics. We structure this section such that we first introduce the simple emission model applied to all emission lines for hydrogen and metals before we discuss some specifics of the complex Ly $\alpha$ emission.

\subsection{General emission model}

There are different mechanisms responsible for the expected extended CGM emission. The first one, referred to as gravitational cooling, is due to the collisional ionization of accreting gas, radiating away part of the energy acquired by compression and shock heating.

The second source is the photoionization by external UV sources, which causes the ionization of the gas and subsequent emission of photons via recombination processes (fluorescence). Among these UV sources, there is the metagalactic UV background (UVB), which consists of the UV photons emitted from distant objects, such as stars or quasars (Haardt \& Madau 2001, 2012; Kollmeier et al. 2014). The computation of the UVBs is a complex task, as many parameters come into play, such as the ionizing photon escape fraction as well as the dust content and opacity and the density distribution of absorbing gas (Haardt \& Madau 2001; Kollmeier et al. 2014; Khaire \& Srianand 2018). In addition to this metagalactic background, there are cases where the presence of a photoionizing bright source nearby, such as a quasar (Cantalupo et al. 2005; Kollmeier et al. 2010; Cantalupo et al. 2014; Martin et al. 2014) enhances the illumination of the gas locally, which then re-radiates through fluorescence.

In the following, we investigate the relative contribution of these different sources to the total luminosity, which is an actively debated topic. We discuss these different mechanisms in the context of our high-resolution simulation and how we take them into account in the post-processing.

The exact determination of the contributions from these different sources requires on-the-fly calculation within the hydrodynamical simulation itself. This has been done for the UV ionizing continuum that impacts the ionization, the temperature and the dynamics of the gas, and consequently changes its emissivity (Rosdahl et al. 2013). A good approximation of this on-the-fly UV ionizing photon transfer has been developed by Rosdahl et al. (2013), namely the 'on-the-fly' self-shielding, used here in our high-resolution simulation.

\subsubsection{Emissivity tables}

Similarly to Bertone et al. (2010) and Frank et al. (2012), we generate emissivity tables for our lines of interest at the corresponding redshifts to attribute a luminosity to each gas cell. These tables account for the flux produced by the gravitational cooling of the gas, and the recombinations from the UVB photoionization. 


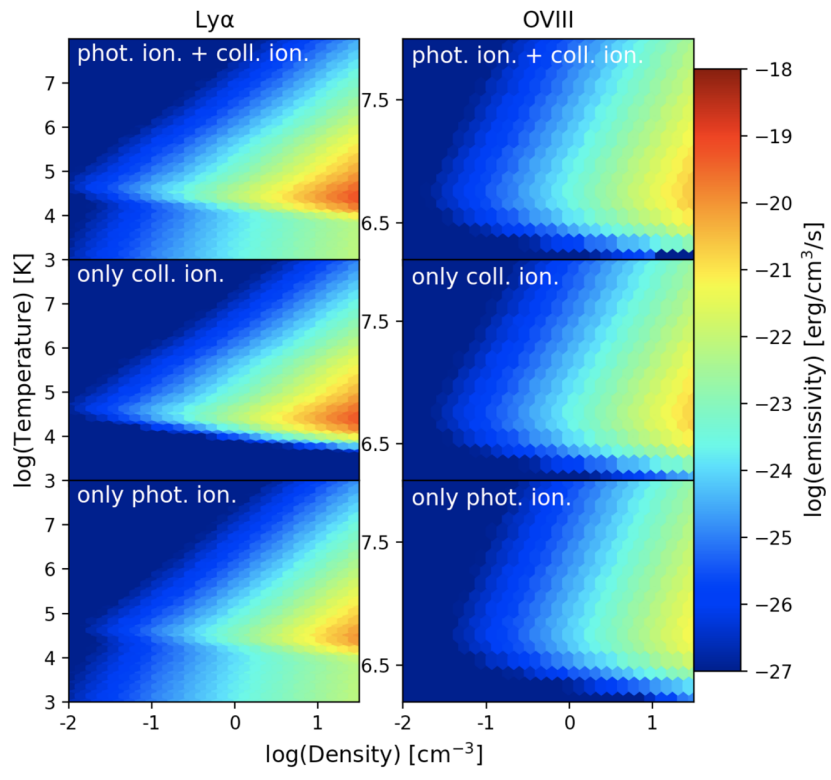

Figure 4. Examples of our CLOUDY emissivity diagrams in units of $\mathrm{erg} \mathrm{s}^{-1} \mathrm{~cm}^{-3}$ in logarithmic scale for Ly $\alpha$ (left) and $\mathrm{O}$ VIII (right) at a redshift of $z=0.33$. The temperature is given in $T / \mu$. The density is given as total hydrogen density in at $\mathrm{cc}^{-1}$. The top panels show the dual contribution of photoionization equilibrium (PIE) and collisional ionization equilibrium (CIE), while the middle panels show the sole contribution of the CIE, used for the self-shielded gas, and the lower panels show the sole PIE contribution, for comparison.

We use the photoionization code CLOUDY, version $10.01,{ }^{2}$ last described by Ferland et al. (1998). This code predicts the thermal, ionization, and chemical structure of a cloud illuminated in a variety of physical conditions. We want to note here that the cooling function in CLOUDY is more sophisticated than the simplistic model for cooling in RAMSES and the inconsistency between the two may introduce a bias in our predictions. The temperature in the simulation will adjust so that the photon emission (especially Ly $\alpha$ ) accounts for the cooling required to roughly balance the total heating rate. Post-processing simulations with inconsistent cooling tables may result in luminosities greater than the heating rates that were present during the simulation. It is however beyond the scope of this paper to investigate this uncertainty further.

We consider a $1 \mathrm{~cm}$ slab of optically thin gas at solar metallicity, ${ }^{3}$ with no molecules and using the element abundances in the solar photosphere as described by Grevesse et al. (2010). In our model, we use the background derived by Haardt \& Madau (2001) (HM01 in the following) with contributions from both quasars and galaxies to be consistent with Frank et al. (2012).

We derive the hydrogen density $n_{\mathrm{H}}=\frac{X_{\odot}}{m_{\mathrm{H}}} \rho$, with $X_{\odot}=0.7380$, and the weighted temperature $T / \mu=\frac{m_{\mathrm{H}}}{k_{\mathrm{B}}} \frac{P}{\rho}$ from the simulation using Grevesse et al. (2010) abundances (also used in the CLOUDY models for consistency).

\footnotetext{
${ }^{2}$ We are using this version of CLOUDY as it includes the option to compile with double floats, which is not computed in the c13 CLOUDY version. This feature is important in our case, as we are deriving the emissivity from low-density regions. These regions can have emissivities below -32 dex.

${ }^{3}$ The emissivity $\epsilon$ scales linearly with metallicity in the first order. We tested this by running several models with varying metallicity, density, and temperature and found a linear correlation between the metallicity and the emissivity on scales between 0.1 and 10 solar metallicities.
}

The tabulation of $\log (T / \mu)$ is done in two steps. First, we generate emissivity tables, as well as electronic density tables in density-temperature $(n, T)$. We use $\log \left(n_{\min }\right)=-8, \log \left(n_{\max }\right)=$ $4, \mathrm{~d}(\log (n))=0.1, \log \left(T_{\min }\right)=2$, and $\log \left(T_{\max }\right)=8, \mathrm{~d}(\log (T))=$ 0.1 . For each point $(n, T)$ in the emissivity tables, we generate a new coordinate $(\log (n), \log (T / \mu))$ using $\mu=\frac{n_{\mathrm{H}} A_{\mathrm{H}}+n_{\mathrm{He}} A_{\mathrm{He}}}{n_{\mathrm{H}}+n_{\mathrm{He}}+n_{\mathrm{e}}}$ where $A_{\mathrm{H}}=$ 1.0074 is the mass number of hydrogen, $A_{\mathrm{He}}=4.002602$ the mass number of helium, and $n_{\mathrm{H}}, n_{\mathrm{He}}$, and $n_{\mathrm{e}}$ are the hydrogen, helium, and electronic density, respectively, tabulated along the emissivity tables. This gives a non-uniformly distributed $(\log (\mathrm{n}), \log (T / \mu))$ emissivity table that we then interpolate back on a regular grid using $\log (T / \mu)_{\min }=\min (\log (T / \mu)), \log \left(T_{\max }\right)=\max (\log (T / \mu))$, and $\mathrm{d}(\log (T / \mu))=\frac{1}{3} \mathrm{~d}(\log (T))$. We then interpolate the different emissivity tables available to the corresponding expansion factor of the considered snapshot.

Fig. 4 shows some examples of the so created emissivity tables for two different lines: Ly $\alpha$ and O VIII. The top panels show the joint contribution of photoionization (PIE) and collisional ionization (CIE), while the middle and lower panels show the sole contribution of CIE and PIE, respectively.

In our model of all the lines, the main contribution to the total emission comes from collisional ionization. Photoionization only plays a role at low temperatures and is negligible in all cases but Ly $\alpha$. Although this is the case in our models, other works find different solutions for the ionization contributions (e.g. Cantalupo 2017; Oppenheimer et al. 2018), suggesting that photoionization is the dominant source for emission from the CGM, rather than collisional ionization.

There are regions in the density-temperature space that are unresolved by CLOUDY, because the occupation of certain states is unlikely and the calculation time intensive. Those are negligible and do not affect our results, as they would show only small emissivity if any. Also, the unresolved regions have generally low temperatures and high densities corresponding to cool ISM gas within the galaxies themselves, away from the CGM regions we are interested in.

\subsubsection{Post-processing self-shielding}

In Section 2.2, a calibrated empirical technique has been used to mimic the effect of self-shielding in the gas temperature and density. Here, we describe how we take the effect of self-shielding into account in the post-processing. Asserting the fraction of gas selfshielded from ionizing radiation is a rather delicate topic. In Frank et al. (2012), the most optimistic self-shielding model uses Popping et al. (2009) results $P / k>258$ and $\tau_{\text {rec }}<\tau_{\mathrm{s}}$, based on the equilibrium between sound speed of the gas and recombination time and an empirical constraint on the thermal pressure. Frank et al. (2012) used this model due to the limiting resolution in their simulation. They present nine different possible choices on the self-shielding thresholds, some of them only including few gas cells from their simulation. With our increased resolution and higher threshold for star formation, we apply a different threshold for the self-shielding than Frank et al. (2012).

We adopt the model proposed by Furlanetto et al. (2004) that simply puts a condition on the temperature (the gas at $T>10^{4.5} \mathrm{~K}$ is collisionally ionized and not self-shielded) and on the density. The density threshold of $n_{\mathrm{HI}} \sim 10^{-2}$ at $\mathrm{cm}^{-3}$ is in line with Rosdahl \& Blaizot (2012) (at $z=3$ ) and Faucher-Giguère et al. (2010) prescription from radiative transfer analysis. Frank et al. (2012) have discussed different cuts for the self-shielding in the post-processing in more detail. The conditions we choose for this work correspond 

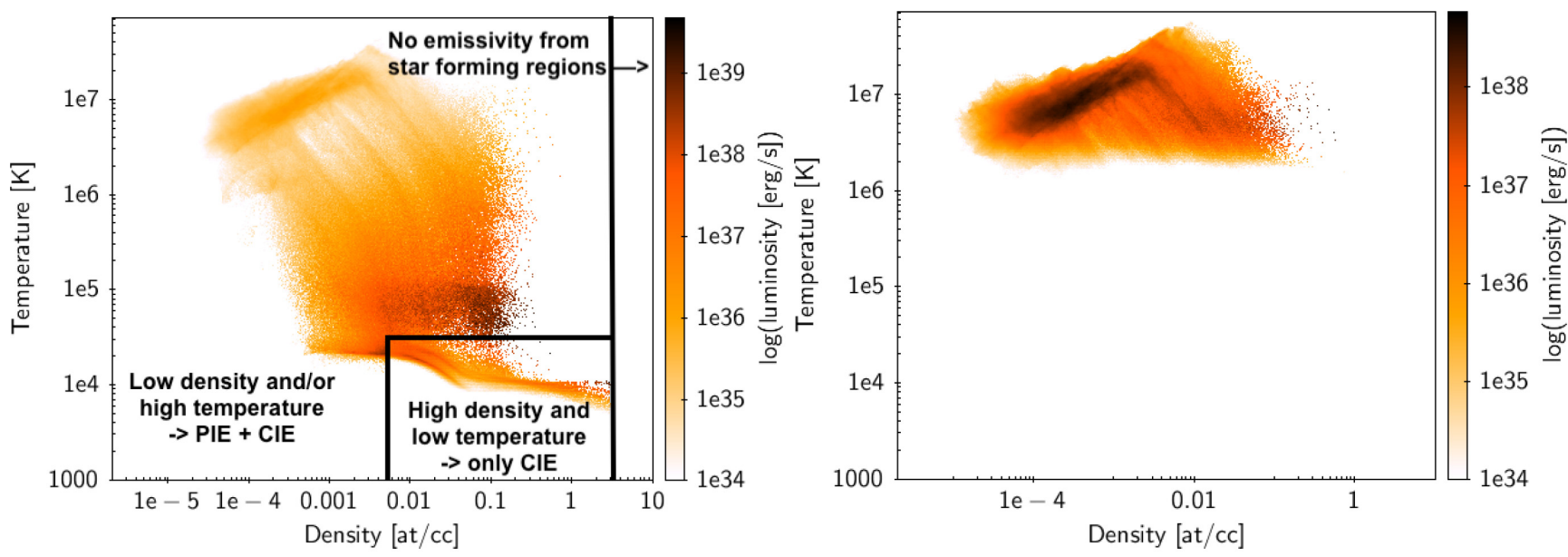

Figure 5. Luminosities resulting from our emissivity predictions applied to gas cells in the selected most massive halo at redshift 0.7 . The density is given as total hydrogen density in at $\mathrm{cc}^{-1}$. The left-hand panel shows the example of Ly $\alpha$. Star-forming regions with a number density of $n_{\mathrm{HI}}>3$ at cc ${ }^{-1}$ are located within the ISM and are not relevant for our CGM emission study. They are therefore assumed to have no emission at all. To account for the self-shielding of gas clouds we apply the cut number 2 from Frank et al. (2012) to identify gas cells for which we only consider collisional ionization (CIE). This leads to null emission from cold regions. For all remaining gas cells, we consider emission from both photoionization (PIE) and collisional ionization (CIE). The right-hand panel shows the emissivity prediction of the same halo for O VIII emission.

to their cut number 2. It considers the three regimes shown in the left-hand panel of Fig. 5. For the ISM region with $n_{\mathrm{HI}}>3$ at $\mathrm{cc}^{-1}$, we consider zero emission. The self-shielded gas with only collisional ionization (CIE) is defined for $n_{\mathrm{HI}}>5.1 \times 10^{-3}$ at cc ${ }^{-1}$ and $T<10^{4.5} \mathrm{~K}$. The rest of the gas emits through both CIE and photoionization (PIE).

\subsubsection{Non-thermal feedback}

For many star-forming galaxy haloes in our high-resolution simulation, we find a smallpercentage $(<1$ per cent) of gas cells with delayed cooling (from the non-thermal feedback). These cells reach temperatures of $10^{5-6} \mathrm{~K}$, with densities consistent with ISM gas cell $\left(n_{\mathrm{H}}>0.1\right.$ at $\left.\mathrm{cm}^{-3}\right)$. They have emissivities of several orders of magnitudes above the value we would expect if their temperature had not been artificially increased, and have therefore also a much higher luminosity than what would be realistic. We have tested the sensitivity of the total halo luminosity against the amount of cells we exclude of the simulations. If we remove more cells than just the ones in delayed cooling, the total luminosity of the halo remains unchanged. If we, however, leave some of the delayed cooling cells in the halo, the luminosity rapidly increases by an order of magnitude. Therefore, we conclude that the exclusion of the delayed cooling cells is a conservative approach.

We choose not to consider these particular cells in the total luminosity budget, as they would in reality not reach these high temperatures but higher pressures, and for shorter time-scales, more as a flash. This consideration brings no particular bias in the total luminosity budget (we remain conservative by not taking them into account), as we checked that these cells, originally associated with ISM gas, should not contribute predominantly to the CGM emission.

\subsection{Special treatment of $\mathrm{Ly} \alpha$}

For Ly $\alpha$ we use the CIE and PIE emission tables just as for the metals. While there is some debate about the dominant source spatially extended Ly $\alpha$ emission (e.g. Cantalupo 2017), collisional ionization is thought to be a main contributor (about 50 per cent of this cooling radiation emerges as Ly $\alpha$ photons) as the photons thus created would be emitted in the dust-poor outskirts of the disc, hence only a small part of these photons is affected by the subsequent immediate dust absorption (Fardal et al. 2001; Dijkstra \& Loeb 2009; Faucher-Giguère et al. 2010).

An additional contribution comes from the production of ionizing photons from star formation or a quasar inside the halo. Indeed, $h v>1$ Ryd photons can ionize the ISM gas, producing photon scattering out of the star-forming regions, although this contribution is mainly Ly $\alpha$ and negligible for metal lines. We take this into account by creating a simple model for the galaxy Ly $\alpha$ emission based on the SFR.

To reproduce the diffuse $\operatorname{Ly} \alpha$ line emission, on-the-fly radiative transfer is not essential as the post-processing of the transfer of the resonant $\operatorname{Ly} \alpha$ photons gives reliable estimates of the total flux emitted (Verhamme, Schaerer \& Maselli 2006; Faucher-Giguère et al. 2010; Kollmeier et al. 2010; Trebitsch et al. 2016).

\subsubsection{Induced processes}

By default, CLOUDY takes into account induced processes: induced recombination and its cooling, stimulated two-photon emission and absorption (Bottorff, Ferland \& Straley 2006), continuum fluorescent excitation, and stimulated emission of all lines (Hazy - a brief introduction to CLOUDY C1O - 1. Introduction and commands, ${ }^{4}$ p. 237). The no induced option turns all these processes off and has also been used in Frank et al. (2012) as well as in Furlanetto et al. (2004, 2005). For a full explanation for this choice we refer the reader to the respective works, but we highlight two of the main reasons:

(i) The absorption and immediate re-emission of isotropically distributed Ly $\alpha$ photons do not contribute to the net luminosity and any excess luminosity due to these processes as calculated in CLOUDY are therefore subtracted from the total emissivity of a gas cell.

${ }^{4}$ https://www.nublado.org/ 
(ii) CLOUDY allows for an unphysical conversion of an absorbed Ly $\beta$ photon ( $1 \mathrm{~s} \rightarrow 3 \mathrm{p})$ to an emitted Ly $\alpha$ photon $(2 \mathrm{p} \rightarrow 1 \mathrm{~s})$ because it assumes mixed orbital angular momentum states for $n \geq 3$. This assumption is motivated for extremely high gas densities $\left(n_{\mathrm{H}} \gg\right.$ $10^{8} \mathrm{~cm}^{-3}$; Pengelly \& Seaton 1964) and therefore not valid in our case.

However, here we explore the actual difference between using induced processes and using the 'no induced processes' option. We note that one of the contributions to the induced processes is the photon pumping or scattering of continuum photons to the Ly $\alpha$ line. This contribution is heavily dependent on the geometry of the gas cloud and therefore likely unconstrained with our simplistic CLOUDY setup. It also depends on nearby ionizing continuum objects, such as young stars or AGN (see e.g. Cantalupo 2017). We rather model the effect from the nearby stars separately (see the next section).

If we run CLOUDY with induced processes and apply it to a simulated halo, the total luminosity in the halo is a factor 2-6 higher than when applying a CLOUDY model with 'no induced processes'. While we choose to use the option 'no induced processes' in our analysis for the above-mentioned reasons and to stay consistent with previous works, this choice will result in a conservative estimate in our predicted halo fluxes.

\subsubsection{Lyo scattering from nearby stellar continuum}

In addition to the photons from the UVB, accounted for in Haardt \& Madau (2001) (HM01), ionizing photons (hv $\geq 1$ Ryd) emitted by nearby young stars, in particular these belonging to the halo in consideration, can contribute substantially to the total Ly $\alpha$ emission of star-forming galaxies. The strength of this contribution depends strongly on the interstellar dust and gas geometry and kinematics (Kunth et al. 2003; Verhamme et al. 2012), as those determine how many Ly $\alpha$ photons escape from the star-forming regions. In a first, conservative approximation, we assume that all the ionizing photons from stars are absorbed by dust or photoionizing neutral gas in the ISM. Since the dust attenuation is poorly constrained at low redshift, we will consider a simplistic model for the emitted Ly $\alpha$ photons. We start with the prescription from Furlanetto et al. (2005) to estimate the intrinsic Ly $\alpha$ luminosity from ionizing photons in the absence of dust: $L_{\text {Ly } \alpha}^{\text {stars }}\left[\mathrm{erg} \mathrm{s}^{-1}\right]=10^{42} \mathrm{SFR}\left[\mathrm{M}_{\odot} \mathrm{yr}^{-1}\right]$. We compute the SFR of each halo from the mass of young stars, using the 'continuous star formation' approximation (Kennicutt 1998):

$\mathrm{SFR}\left[\mathrm{M}_{\odot} \mathrm{yr}^{-1}\right]=\frac{M_{\text {stars }<10^{8} \mathrm{yr}}\left[\mathrm{M}_{\odot}\right]}{10^{8}[\mathrm{yr}]}$.

Using COS data of low-redshift $(z \sim 0.03)$ star-forming galaxies, Wofford, Leitherer \& Salzer (2013) measured a Ly $\alpha$ escape fraction ranging from 1 percent to 10 per cent. They estimate that this fraction is sensitive to the presence of dust and to the H I column density, the Ly $\alpha$ photons escaping more easily from holes of low H I and dust column densities, resulting in a large scatter. Winds can also have a strong effect and can help Ly $\alpha$ photons to escape (Dijkstra \& Jeeson-Daniel 2013) but we do not consider winds in our model. As we do not have any model for either the dust or radiative transfer, we will stay conservative in our assumptions. Hayes et al. (2011) find $\operatorname{Ly} \alpha$ escape fractions between 0.1 per cent and 1 per cent for low- $z$ galaxies. Therefore, we will consider two extreme cases for the Ly $\alpha$ luminosity from the stellar contribution at low redshift: one with a Ly $\alpha$ escape fraction of 1 per cent, and another with a Ly $\alpha$ escape fraction of 0.1 per cent. At higher redshift $(z>1)$, we adopt a Ly $\alpha$ escape fraction of 10 per cent that is within the prediction of
Hayes et al. (2011). We are aware that different works predict very different escape fractions for $\operatorname{Ly} \alpha$ (e.g. Wofford et al. 2013; Naidu et al. 2017, but we choose to follow the trend observed in Hayes et al. (2011). Predicting the spatial and spectral profiles of such emission would require the full calculation from radiative transfer techniques (Verhamme et al. 2006, 2012; Rosdahl et al. 2013; Lake et al. 2015), which is beyond the scope of this work. However, as our goal is to study the detectability of such emission with upcoming instruments, we chose to make the simple assumption that all the Ly $\alpha$ photons only go through one absorption/re-emission process before leaving the cloud. Also, we assume that all of the Ly $\alpha$ photons are emitted from the centre of the galaxy. We then weigh the profile proportionally to the total hydrogen density of the gas cell and by its inverse squared distance to the centre. This gives us, for each cell $j$ the luminosity $L_{j}^{\star}$ :

$L_{j}^{\star}\left[\operatorname{erg~s}^{-1}\right]=f_{\mathrm{esc}}(\mathrm{Ly} \alpha) \frac{\frac{n_{j, \mathrm{H}}}{R_{j}^{2}}}{\int_{R_{\mathrm{vir}}} \frac{n_{\mathrm{H}}}{R^{2}}} 10^{42} \mathrm{SFR}_{j}\left[\mathrm{M}_{\odot} \mathrm{yr}^{-1}\right]$.

\subsubsection{Total Lya luminosity}

As described in previous sections, we consider collisional ionization (gravitational cooling) and photoionization from UVB photons.

We consider that the total luminosity for the $\operatorname{Ly} \alpha$ line is the sum of these two quantities:

$L_{\mathrm{Ly} \alpha}^{\mathrm{total}}=L_{\mathrm{Ly} \alpha}^{\text {grav.cooling+UVBfluo. }}+L_{\mathrm{Ly} \alpha}^{\text {stars }}$.

This consideration is not completely realistic, as we should strictly take into account the ionizing flux from the young stars combined with the UVB used in the CLOUDY model and during the simulation computation to reproduce the density/temperature state of the gas in these conditions. The RAMSES simulation used in this work only reproduces the gravitational effects and the heating from the UVB. Regarding the purpose of this work, this assumption should be accurate enough to give valuable insights on the level of radiation from the CGM.

\subsection{UV continuum}

To properly reproduce mock observations in the UV, we now need to model the UV continuum of each halo. We first compute the SFR of each halo from the mass of young stars (see equation 1), from which we infer the flux derived by Kennicutt (1998), $L(\lambda)\left[\mathrm{erg} \mathrm{s}^{-1}\right]=$ $\frac{\mathrm{SFR}\left[\mathrm{M}_{\odot} \mathrm{yr}^{-1}\right]}{1.4} \frac{c}{\lambda^{2}} 10^{28}$. To derive the spatial extent of this continuum, we assume that the UV continuum is mainly produced by these young stars, so we use the stars whose age is less than $10^{8} \mathrm{yr}$ to derive a 'stellar density field' that we scale with $L_{\lambda, 1500 \AA}(\lambda)$ :

$L_{\lambda, 1500 \AA}(\lambda)\left[\mathrm{erg} \mathrm{s}^{-1}\right]=10^{-0.4 k^{\prime} E(B-V)} \frac{\mathrm{SFR}\left[\mathrm{M}_{\odot} \mathrm{yr}^{-1}\right]}{1.4} \frac{c}{\lambda^{2}} 10^{28}$.

To account for the dust attenuation of these continuum photons, we use the model from Zahid et al. (2012) to get the colour excess $E(B-V)$ from the sum of the stellar particles (not just the young stars) using their mass $M_{\star}$ and metallicity $Z$ :

$E(B-V)=0.44\left(p_{0}+p_{1} Z^{p_{2}}\right) M^{p_{3}}$,

where $\quad Z=10^{(12+\log (\mathrm{O} / \mathrm{H})-8)}, M=M_{\star} / 10^{10}, p_{0}=0.12 \pm$ $0.01, p_{1}=0.041 \pm 0.006, p_{2}=0.77 \pm 0.06$, and $\quad p_{3}=$ $0.240 \pm 0.002$. This model from Zahid et al. (2012) has been fitted to SDSS data to determine those parameters. The fit rms is $\sim 0.11$ dex. Particularly, the high stellar mass-high 


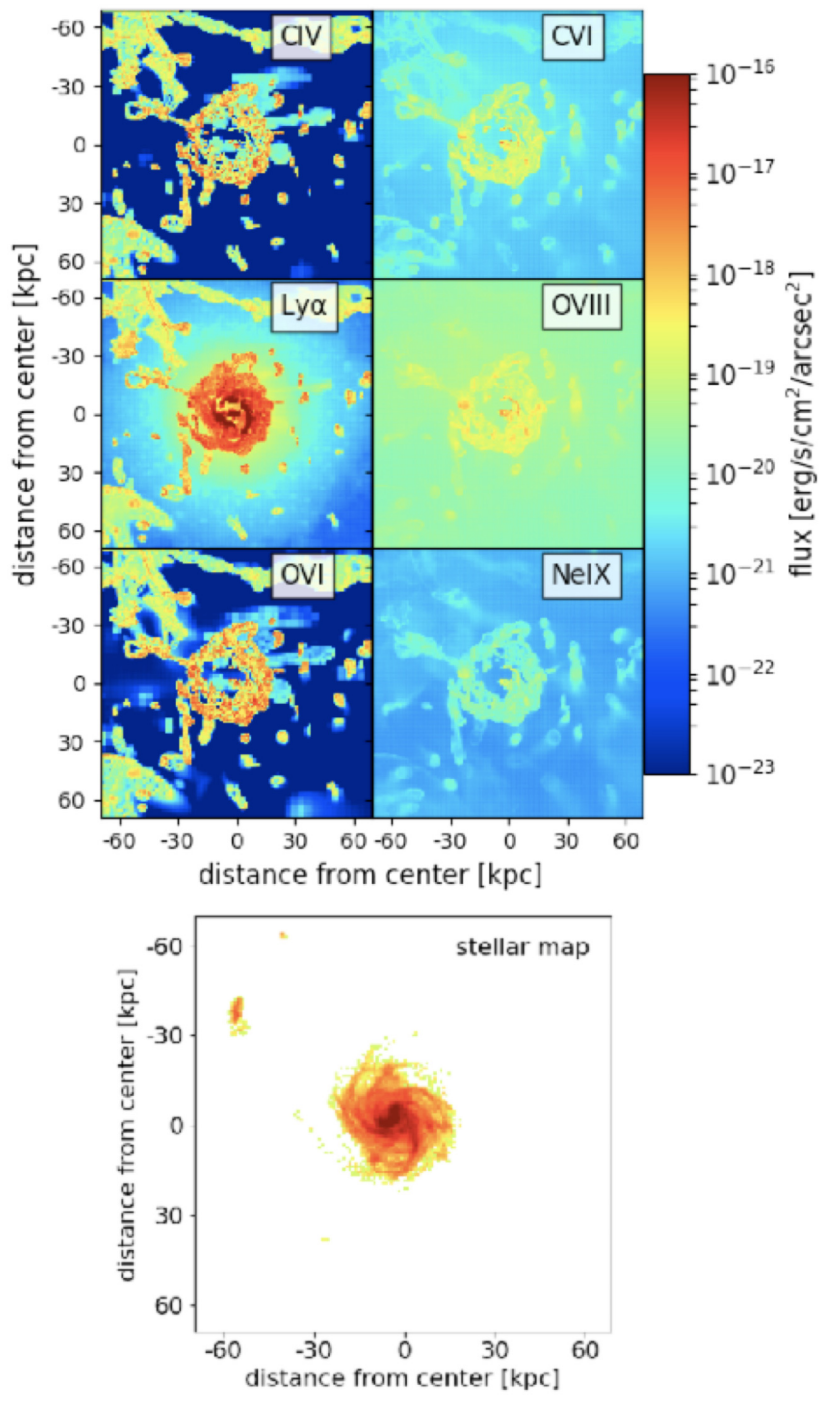

Figure 6. Upper panel: Simulated flux emission predictions of various lines at redshift 0.3 . Pixel scale is 0.2 arcsec corresponding to at the given redshift. We find Ly $\alpha$ to be the brightest emission line among the lines emitting at UV wavelengths (left-hand panels) and O VIII to be the brightest among the $\mathrm{X}$-ray lines (right-hand panels). We show all lines with the same colour scale to illustrate the distribution of the different gas phases in the CGM. The emission from colder gas (lower ionization states) comes from clumpy structures in the CGM with only little flux from the areas in between. The hot component, traces the same structures but with a much higher flux level also in between the individual dense gas clumps, such that a more homogeneous distribution of flux over the entire halo becomes apparent. See also Fig. A4 in the appendix for a comparison between $\mathrm{Ly} \alpha$ and $\mathrm{O}$ VIII emission on larger scales. Lower panel: Stellar map of the most massive halo at redshift 0.3 . metallicity, and therefore high colour excess, objects from the SDSS observations are slightly underpredicted in their work, which may affect our mock observations such that the colour excess for the most massive haloes is slightly underestimated. We consider the stellar mass weighted metallicity to derive $\mathrm{Z}$ from the simulation. We derive the extinction $k^{\prime}(\lambda)$ following Calzetti et al. (2000):

$k^{\prime}(\lambda)=2.659\left(-2.156+1.509 / \lambda-0.198 / \lambda^{2}+0.011 / \lambda^{3}\right)+R_{V}^{\prime}$

for $0.12 \mu \mathrm{m} \leq \lambda \leq 0.63 \mu \mathrm{m}$. We choose to use $R_{V}^{\prime}=3.1$ to account for a dusty environment such as the Galactic diffuse ISM. The attenuation of the continuum then scales as $10^{-0.4 k^{\prime} E(B-V)}$. We make the assumption that the attenuation is homogeneous within the ISM across the projected image of its UV continuum.

\section{RESULTS}

In this section, we present the results of our simulations and compare them to observations in order to estimate how realistic our predictions are. We note that the main aspect of our work is the emission prediction of galaxy haloes rather than the overall properties of the cosmological simulation itself.

\subsection{Different ions in the most massive halo at $z=0.3$}

Once we apply the emission prediction model to galaxy haloes from the simulation, we can calculate the luminosity and the flux in each cell for a given ion at a given wavelength. We use these calculated fluxes to create data cubes of these haloes that represent mock observations with two spatial axes and one spectral axis. First of all we look at the qualitative difference of emission from different ions from the most massive halo at a given redshift. We consider the most massive halo at redshift 0.3. Fig. 6 shows the outcome of the emission prediction for the most massive halo at different wavelengths, corresponding to different ions. We see that the gas emitting in Ly $\alpha, \mathrm{C}$ IV, and O VI lines (left-side panels) is much more concentrated in clumps than the hot gas emitting C VI, O VIII, and $\mathrm{Ne}$ IX lines on the right-side panels that seem more homogeneously distributed around the central galaxy. We also find that Ly $\alpha$ is the overall brightest line amongst the UV lines and O VIII the most promising X-ray line from high-temperature gas.

\subsection{Comparison of the most massive halo luminosity to the low-resolution simulation near $z \sim 0.67$}

We note that the maximum Ly $\alpha$ luminosity at low redshifts (see Table 4) does not exceed a few $10^{42} \mathrm{erg} \mathrm{s}^{-1}$. Yet, our high-resolution simulation is based on one of the brightest and most massive haloes from the analysis performed by Frank et al. (2012). In their analysis for $\operatorname{Ly} \alpha$ at $z \sim 0.67$, they predict $\operatorname{Ly} \alpha$ luminosities to go up to

Table 4. Most massive halo luminosity and integrated flux predictions from our simulations for different ions and redshifts for a given Ly $\alpha$ escape fraction.

\begin{tabular}{|c|c|c|c|c|c|c|c|c|c|}
\hline$z$ & $\begin{array}{c}f_{\text {esc }} \\
\text { (per cent) }\end{array}$ & $\begin{array}{c}L_{\mathrm{Ly} \alpha} \\
\left(10^{42} \mathrm{erg} \mathrm{s}^{-1}\right)\end{array}$ & $\begin{array}{c}\log \left(f_{\text {Ly } \alpha}\right) \\
\left(\operatorname{erg~s}^{-1} \mathrm{~cm}^{-2}\right)\end{array}$ & $\begin{array}{c}L_{\mathrm{CIV}} \\
\left(10^{42} \mathrm{erg} \mathrm{s}^{-1}\right)\end{array}$ & $\begin{array}{c}\log \left(f_{\text {CIV }}\right) \\
\left(\operatorname{erg~s}^{-1} \mathrm{~cm}^{-2}\right)\end{array}$ & $\begin{array}{c}L_{\mathrm{OVI}} \\
\left(10^{42} \mathrm{erg} \mathrm{s}^{-1}\right)\end{array}$ & $\begin{array}{c}\log \left(f_{\mathrm{OVI}}\right) \\
\left(\operatorname{erg~s}^{-1} \mathrm{~cm}^{-2}\right)\end{array}$ & $\begin{array}{c}L_{\mathrm{O} \text { VIII }} \\
\left(10^{42} \mathrm{erg} \mathrm{s}^{-1}\right)\end{array}$ & $\begin{array}{c}\log \left(f_{\text {O VIII }}\right) \\
\left(\operatorname{erg~s}^{-1} \mathrm{~cm}^{-2}\right)\end{array}$ \\
\hline 4.0 & 10 & 10.0 & -16.2 & 0.71 & -17.4 & 0.92 & -17.2 & 0.05 & -18.5 \\
\hline 2.3 & 10 & 35.9 & -15.1 & 1.17 & -16.6 & 1.44 & -16.5 & 0.35 & -17.1 \\
\hline 1.0 & 1 & 7.93 & -14.8 & 1.56 & -15.5 & 1.49 & -15.6 & 0.64 & -15.9 \\
\hline 0.67 & 1 & 3.73 & -14.7 & 1.16 & -15.2 & 1.16 & -15.5 & 0.47 & -15.6 \\
\hline 0.25 & 0.1 & 2.14 & -13.9 & 1.18 & -14.2 & 1.17 & -14.2 & 0.51 & -14.6 \\
\hline
\end{tabular}




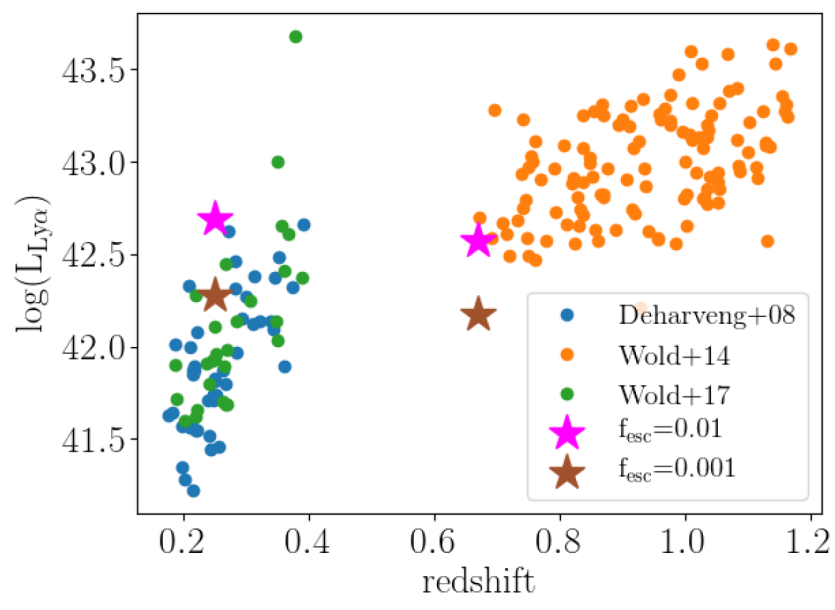

Figure 7. Comparison of the predicted Ly $\alpha$ luminosity at low redshifts with GALEX observations for the most massive halo as described in Table 3 and different Ly $\alpha$ escape fractions. We plot the most massive halo and so one of the most luminous. We adjust the escape fraction such that this most massive halo fits well into the observations, which are also the most luminous ones.

$10^{44} \mathrm{erg} \mathrm{s}^{-1}$, without even accounting for the SFR induced Ly $\alpha$ luminosity. This two dex difference finds its origin in the recipe used for the high-resolution simulation. Indeed, while we used 'on-thefly' self-shielding in our simulation, preventing $n_{\mathrm{H}}>10^{-2}$ at cc ${ }^{-1}$ gas cell to be heated by the metagalactic UVB, the gas cells in Frank et al. (2012) show larger temperature in the cooling 'gutter' of Ly $\alpha$ emission (see Fig. 3). Specifically, we find the temperature in our cooling gutter at $n=0.03$ at cc$^{-1}$ around $10^{4} \mathrm{~K}$, while the temperature in the cooling gutter in the low-resolution simulation at the same density is around $2 \times 10^{4} \mathrm{~K}$. This low increase of the equilibrium temperature has dramatic effects on the effective emission rate, as there is a steep evolution of the Ly $\alpha$ cooling emissivity with temperatures of a few $10^{4} \mathrm{~K}\left(10^{2} \epsilon\left(10^{4} \mathrm{~K}\right) \approx \epsilon(2 \times\right.$ $\left.10^{4} \mathrm{~K}\right)$ ), see fig. 6 in Rosdahl \& Blaizot (2012). We therefore argue that the emission level predicted in our model is more realistic, despite being less optimistic, than those derived in the original study as the crucial question of cooling temperature has been optimized since the last implementation of the code.

\subsection{Validation with low-redshift observations}

We want to see how well our simulations compare to actual observations at low redshift. This is important not only to verify how realistic the simulations are but also to see how well they are suited for the preparation of future observations with upcoming UV instruments. For this exercise we compare the simulated flux in the most massive haloes at redshift 0.3 and 0.67 with GALEX observations of Ly $\alpha$ emitting galaxies (Deharveng et al. 2008; Wold, Barger \& Cowie 2014; Wold et al. 2017). Since the Ly $\alpha$ escape fraction at low redshifts can vary between 0.1 per cent and 1 per cent we consider both of these escape fractions (Hayes et al. 2011). Fig. 7 shows this comparison. For redshift $z>0.5$ we are generally underestimating the observed flux, although our simulations are still in agreement with observations for $f_{\text {esc }}=1$ per cent. For the lowest redshifts $(z<0.5)$, we are slightly overpredicting the flux and the flux for $f_{\text {esc }}=0.1$ per cent is only marginally in agreement with the observations. The most massive halo in our simulation, chosen due to its high resolution $(380 \mathrm{pc})$, is one of the most luminous due to its mass and size. The GALEX observations are, due to the instrument's detection limit, the most UV luminous galaxies at low redshifts, while there probably are many more less luminous galaxies at these redshifts. In this context we conclude that our simulations are in agreement with the observed Ly $\alpha$ luminosities at low redshifts. Given the results of this comparison we choose a Ly $\alpha$ escape fraction of $f_{\mathrm{esc}}=0.1$ per cent for $z<0.5$ and $f_{\mathrm{esc}}=1$ per cent for $0.5<z \leq 1.0$.

\subsection{Comparison to high-redshift observations}

We consider recent observations of $\mathrm{Ly} \alpha$ emission from high-redshift Ly $\alpha$ haloes from the Subaru telescope $(z=2.3$, Momose et al. 2014) and VLT/MUSE ( $z=4.0$, Wisotzki et al. 2016) to validate our model. Although the model described in the previous sections is originally set up such that it represents low-redshift $(z \leq 1)$ objects, we use the same prescription for higher redshifts. We test it at two example redshifts: $z=2.3$ and $z=4.0$. Using this model for higher redshifts does not bring major changes in the post-processing selfshielding treatment, as the density cut has been calibrated from high-redshift simulated galaxies (Rosdahl \& Blaizot 2012; see also Katz et al. 1996; Schaye 2001) and the temperature cut is purely empirical. The dust attenuation calculation for the continuum is also considered redshift independent as the properties of dust grains should not evolve much. However, the Ly $\alpha$ escape fraction $f_{\mathrm{esc}}^{\mathrm{Ly} \alpha}$ is redshift dependent, and can be of the order of 10 per cent at redshift 4 (Hayes et al. 2011).

We note here that the comparison is a simplified case study of single objects. This is due to scarcity of observations of galaxies with the properties that we require to do a meaningful comparison. However, this single object case study is appropriate to estimate the reliability of our simulations.

\subsubsection{Surface brightness profiles at $z=2.2$}

The surface brightness (SB) profile at $z=2.2$ performed by Momose et al. (2014) consists in the stacking of 3556 LAEs, the comparison to one of our objects is therefore only illustrative. We identify a halo with $M_{\star}=4.8 \times 10^{10} \mathrm{M}_{\odot}$ and SFR $=91.9 \mathrm{M}_{\odot} \mathrm{yr}^{-1}$ in the simulation, which reproduces a continuum level similar to that of the stack. The top left panel of Fig. 8 shows the SB map of the continuum of the selected object, while the bottom left panel shows its SB radial profile with a comparison the stack. As in the analysis by Momose et al. (2014), we convolved the image with a PSF of 1.32 arcsec FWHM to reproduce the largest seeing size of the stacked images. The top right panels shows the SB map of the selected object Ly $\alpha$ line, with the same convolution than the continuum. The bottom right panel shows the SB radial profile for the simulated object using Ly $\alpha$ escape fractions of $\{0,0.3,3\}$ per cent and that of the stack. For $R<1.5$ arcsec, we are able to reproduce the Ly $\alpha$ line level with a Ly $\alpha$ escape fraction of 0.3 per cent (which is ten times below the prescription from Hayes et al. (2011) at this redshift), which corresponds to a Ly $\alpha$ luminosity of $L_{\mathrm{Ly} \alpha}=3.46 \times 10^{41} \mathrm{erg} \mathrm{s}^{-1}$.

We associate this underestimation of the $\operatorname{Ly} \alpha$ escape fraction at small radii with the uncertainties in the simulation and the uncertainties in the estimation of the Ly $\alpha$ escape fraction itself (Hayes et al. 2011). Also the effect of stacking in Momose et al. (2014) as well as the SFR of the chosen halo (see Matthee et al. 2016) can play a role in this discrepancy. We conclude overall that our simulated profile is comparable to observations.

At higher radii $(r \gtrsim 15 \mathrm{kpc}$ ) there seems to be an offset, which could mean an underprediction of the CGM flux in our simulation. 

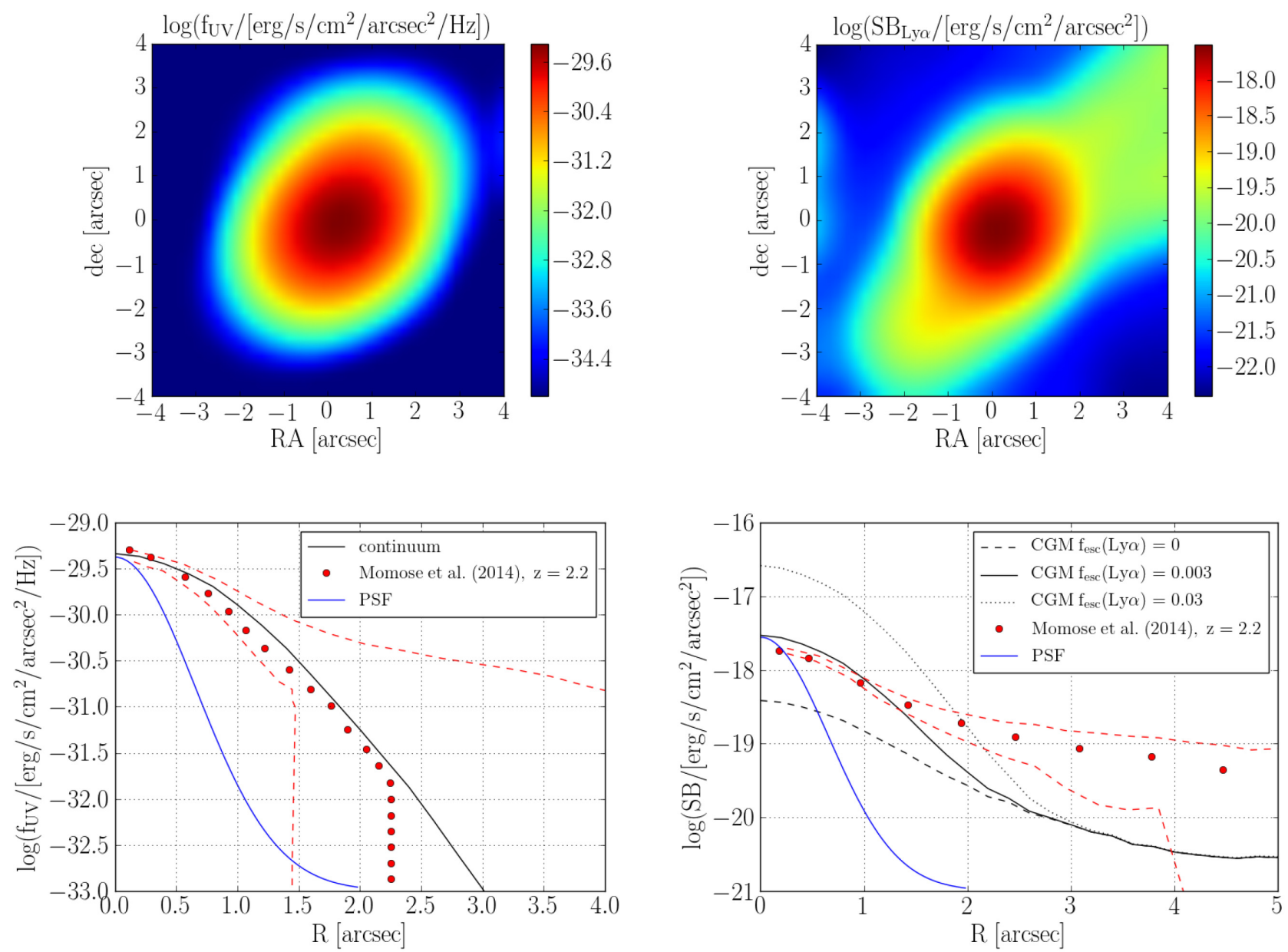

Figure 8. Top left panel: Surface brightness map of the continuum of the halo selected for the comparison with the stack of 3556 LAEs from Momose et al. (2014) at $z=2.2$. Bottom left panel: SB radial profile of the continuum for the selected halo and the stack. Top right panel: Surface brightness map of the Ly $\alpha$ line emission, using $f_{\mathrm{esc}}(\mathrm{Ly} \alpha)=0.003$, for the halo selected in our study. Bottom right panel: SB radial profile of the Ly $\alpha$ line emission for the selected halo with Ly $\alpha$ escape fractions of $\{0,0.3,3\}$ per cent and for the stack. The observed halo has a brighter CGM from $R=1.5$ arcsec onwards, although the observed data points also become noisier in those outskirts. The red dashed lines give the uncertainty envelope on the observations within $1 \sigma$. At the considered redshift of 2.2, 1 arcsec corresponds to $8.4 \mathrm{kpc}$. The blue curve shows the shape of the PSF (FWHM=1.32 arcsec) with which we convolved our simulated data.

This would not be surprising, given that we do not use AGN feedback that is expected to drive more matter outside of the galaxy into the CGM. Also the lack of cosmic rays in our simulation may have a role in this discrepancy. Hopkins et al. (2019) have recently shown that cosmic rays can have a significant impact on the CGM, especially at radii of $\mathrm{r} \gtrsim 200 \mathrm{kpc}$ as they keep cool gas from raining on to the galaxy. Yet, the observations at these larger radii are relatively uncertain and are not sufficient to draw a strong conclusion at this point. For our purposes, the CGM flux is reproduced well enough in our simulations.

\subsubsection{Surface brightness profiles at $z=4$}

We chose the object \#308 from Wisotzki et al. (2016) for the comparison, as it lies at a redshift matching our high-resolution simulation set. We select a halo from the simulation based on the continuum SB profile that reproduces the continuum level of the object. The selected halo has a stellar mass $M_{\star}=7.0 \times 10^{9} \mathrm{M}_{\odot}$ and an SFR of $50.9 \mathrm{M}_{\odot} \mathrm{yr}^{-1}$, which is slightly above the prescription from Wisotzki et al. (2016) $\left(M_{\star}=10^{8-9} \mathrm{M}_{\odot}\right.$ and $\left.\mathrm{SFR}=0.3-16 \mathrm{M}_{\odot} \mathrm{yr}^{-1}\right)$. The top left panel of Fig. 9 shows the
SB map of the continuum of the selected halo. We convolved the image with a 0.66 arcsec FWHM PSF to account for the seeing and with a 0.71 arcsec FWHM PSF to reproduce the instrument's resolution (Bacon et al. 2014). The bottom left panel shows the SB radial profile for the selected halo and object \#308. The top right panel shows the SB map of the Ly $\alpha$ line for the selected halo, with the same convolutions as the continuum. The bottom right panel shows the SB radial profile for the simulated object using Ly $\alpha$ escape fractions of $\{0,2,10\}$ per cent and that of object \#308. We recover a similar Ly $\alpha$ luminosity than the one measured by Wisotzki et al. (2016) for object \#308 $\left(L_{\mathrm{Ly} \alpha}=1.6 \times 10^{42} \mathrm{erg} \mathrm{s}^{-1}\right)$ with $f_{\mathrm{esc}}(\mathrm{Ly} \alpha)=2$ per cent: $L_{\mathrm{Ly} \alpha}=2.0 \times 10^{42} \mathrm{erg} \mathrm{s}^{-1}$. This Ly $\alpha$ escape fraction is a few times lower than what Hayes et al. (2011) find at this redshift but we accept this difference as both the profiles as well as the Ly $\alpha$ escape fraction determination have some uncertainties associated. Again, at larger radii $(R>1.5 \operatorname{arcsec})$, the simulated profile is lower than the observed one. We note here that while the deepest MUSE observations can reach a detection limit of $2.8 \times 10^{-20} \mathrm{erg} \mathrm{s}^{-1} \mathrm{~cm}^{-2} \operatorname{arcsec}^{-2} \AA^{-1}$ (Leclercq et al. 2017), the specific observations we compare with reach their detection limit at $10^{-19} \mathrm{erg} \mathrm{s}^{-1} \mathrm{~cm}^{-2} \operatorname{arcsec}^{-2}$ and are therefore not easily comparable to the simulations at large radii. 

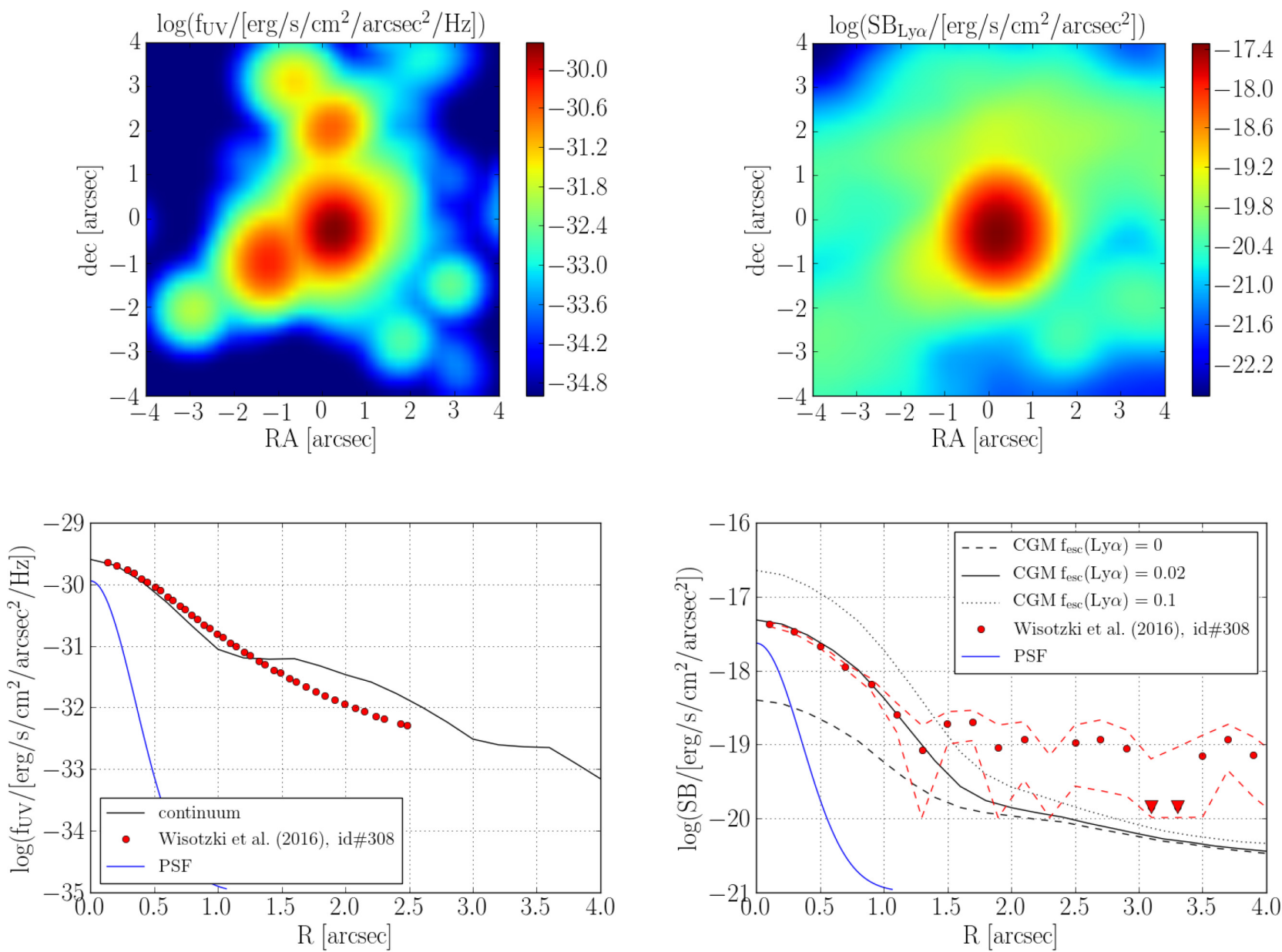

Figure 9. Top left panel: Surface brightness map of the continuum of the halo selected for the comparison with object \#308 from Wisotzki et al. (2016) at $z=4.018$. Bottom left panel: SB radial profile of the continuum for the selected halo and object \#308. Top right panel: Surface brightness map of the Ly $\alpha$ line emission, using $f_{\mathrm{esc}}(\mathrm{Ly} \alpha)=2$ per cent for the selected halo. Bottom right panel: SB radial profiles of the Ly $\alpha$ line emission for the selected halo with Ly $\alpha$ escape fractions of $\{0,2,10\}$ per cent and for object \#308. The detection limit of the observed data is around $\log (\mathrm{SB}) \sim-19$. The red dashed lines give the uncertainty envelope on the observations within $1 \sigma$ and are statistically consistent with our predictions given the assumptions in the model. At the considered redshift of 4.0, 1 arcsec corresponds to $7.1 \mathrm{kpc}$. The blue curve shows the shape of the PSF (FWHM $=0.66$ arcsec) with which we convolved our simulated data.

\section{LOW-REDSHIFT ( $Z \sim 0.7)$ UV OBSERVATIONS WITH FIREBALL-2}

FIREBall-2 (PI: Chris Martin; Milliard et al. 2010; Picouet et al. 2018) is a balloon-borne experiment aiming at observing the faint diffuse UV emission from the CGM of intermediate-redshift (0.31.0) galaxies. It consists in a UV Multi-Object slit Spectrograph (MOS) with a resolution of $R \sim 2000$, and an FWHM of $\sim 6$ $\operatorname{arcsec}$ over an effective field of view of $37 \times 20 \operatorname{arcmin}^{2}$. It is optimized to observe in a narrow wavelength range, 199-213 nm. This wavelength range corresponds to the 'sweet spot' of dioxygen and ozone atmospheric absorption. FIREBall was launched in September 2018 from Fort Sumner, New Mexico, targetting Ly $\alpha$ emission from $z \sim 0.7$ galaxies, O VI emission from $z \sim 1$ galaxies and CIV emission from $z \sim 0.3$ galaxies. We summarize the relevant characteristics of the instrument in Table 5. FIREBall is pathfinder experiment for a more ambitious project, ISTOS (PI: C. Martin; Martin 2014), a UV IFS satellite to be proposed to NASA.
Table 5. FIREBall-2 instrument specifications: We summarize here the critical characteristics of the FIREBall-2 instrument. These are also the instrument model parameters that directly impact our SNR analysis.

\begin{tabular}{|c|c|}
\hline Parameter & Value \\
\hline Spectral resolution & $\sim 2000 \lambda / \delta \lambda$ \\
\hline FWHM & $\sim 5-6 \operatorname{arcsec}$ \\
\hline Effective field of view & $37 \times 20 \operatorname{arcmin}^{2}$ \\
\hline Wavelength range & $199-213 \mathrm{~nm}$ \\
\hline Diameter of mirror & $1 \mathrm{~m}$ \\
\hline Number of objects observable per night & $\sim 200-300$ with $2 \mathrm{~h}$ exposure \\
\hline Sky background & 500 photons s ${ }^{-1} \mathrm{~cm}^{-2} \mathrm{sr}^{-1} \AA^{-1}$ \\
\hline Acquisition time per field & $2 \mathrm{~h}$ \\
\hline Dark current & $0.036 \mathrm{e}^{-}$pixel $^{-1} \mathrm{~h}^{-1}$ \\
\hline Induced charge & $0.002 \mathrm{e}^{-}$pixel $^{-1}$ frame $^{-1}$ \\
\hline Read noise & Negligible in photon counting mode \\
\hline Detector effective QE & $\sim 55$ per cent \\
\hline Total optical throughput & 13 per cent \\
\hline Atmospheric throughput & 55 per cent \\
\hline
\end{tabular}




\subsection{Instrument model}

In order to prepare for the upcoming data analysis of FIREBall2, Mège et al. (2015) developed a code that simulates the endto-end image reconstruction process along the optical path of the instrument. This code, coupled to ZEMAX, generates a set of point spread functions (PSFs) from an optical model at any given field positions and wavelengths. These PSFs are then interpolated at any point (in the field and wavelength), giving access to fundamental optical properties (magnification matrix, optical throughput, optical distortion, spectral dispersion) derived from the optical mappings existing between the sky plane and the instrument's mask or detector plane. Secondly, it produces 2D images of the electronic map of a detector patch corresponding to the observation of a modelled emission line from a simulated galaxy. Since its implementation in 2015 the code has constantly been modified according to the changes and updated measurements on the FIREBall instrument itself. We use the instrument specific values given in Table 5 for our calculation with the instrument model.

In the following, we combine the emission prescription to the instrument model to perform an end-to-end analysis of the observation of the CGM of low-redshift galaxies with the MOS of FIREBall-2.

\subsection{Predicted signal with FIREBall observations}

The total image is a Poisson realization of the additive contribution of the CGM emission, the galaxy disc line emission, the continuum of the galaxy $(\widehat{\mathrm{GAL}})$, the sky $(\widehat{\mathrm{SKY}})$, the dark current from the detector ( $\widehat{\mathrm{DARK}})$ and an induced charge current from the detector $\widehat{(\mathrm{CIC})}$. We use estimators for these contributions. When observing an emission line from a galaxy halo, there are two contributions: the galaxy itself and the CGM. There is no physical motivated border between the two, so for our further analysis we will call the combination 'extended line emission' ( $\widehat{\mathrm{ELE}})$. Consequently the 'MeasuredSignal' is the sum of all contributions mentioned above and the signal we are interested in is the following:

$\widehat{\mathrm{ELE}}=$ MeasuredSignal $-\widehat{\mathrm{GAL}}-\widehat{\mathrm{SKY}}-\widehat{\mathrm{DARK}}-\widehat{\mathrm{CIC}}$.

The dark current is known from the calibration of the detector to be $\widehat{\text { DARK }}=0.0036 \mathrm{e}^{-}$pixel $^{-1} \mathrm{~h}^{-1}$ at $-110^{\circ} \mathrm{C}$ and a negligible variance due to an estimate on a large number of pixels with respect to the dominant noise source that is the galaxy continuum. We first remove the dark from the signal. We then estimate the profile of the continuum from regions towards the end of the galaxy spectrum, which are free of emission lines, $P_{x, 1}$ and $P_{x, 2}$, by stacking the columns of pixels (without the dark) over $\sim 10$ columns in the dispersion direction. $x$ and $\lambda$ give the spatial and spectral coordinate on the detector. The resulting continuum estimate is then an interpolation via linear regression between the two regions:

$\widehat{\operatorname{GAL}}_{x, \lambda}=(1-\alpha) P_{x, 1}+\alpha P_{x, 2}$,

$\lambda_{1}$ and $\lambda_{2}$ are the central wavelengths of each region and $\alpha=\frac{\lambda-\lambda_{1}}{\lambda_{2}-\lambda}$. The corresponding variance is

$\sigma_{\overline{\mathrm{GAL}}_{x, \lambda}}^{2}=(1-\alpha)^{2} \sigma_{P_{x, 1}}^{2}+\alpha^{2} \sigma_{P_{x, 2}}^{2}$.

Considering a Poissonian distribution for the photon noise, the variance on the measurement is computed as the image $\sigma_{x, \lambda, \text { meas }}^{2}=d_{x, \lambda}$. We neglect the read out noise as we use the detector in counting mode. The last contribution to our SNR estimation comes from the induced charge of the detector, which is assumed a noiseless con- stant, $\widehat{\mathrm{CIC}}$. Any other sources of noise are considered negligible. As the current pixel size on the detector oversamples the resolution, we need to consider the contribution of a detector area corresponding to the actual resolution element. We therefore compute an SNR per resolution element by convolving the continuum-subtracted signal (and the corresponding noise) with the estimator for the instrument PSF ( $\widehat{\mathrm{PSF}})$, normalized by the maximum pixel value. The SNR per resolution element then becomes

$$
\mathrm{SNR}_{x, \lambda}^{\mathrm{PRE}}=\frac{((d-\widehat{\mathrm{DARK}}-\widehat{\mathrm{GAL}}-\widehat{\mathrm{CIC}}) * \widehat{\mathrm{PSF}})_{x, \lambda}}{\sqrt{\left(\left(d+\sigma_{\widehat{\mathrm{GAL}}}^{2}\right) * \widehat{\mathrm{PSF}}\right)_{x, \lambda}}} .
$$

In order to mock real observations we need to add some noise. Therefore, we use a Poissonian realization of the analytic solution of the SNR. From this we can then infer the maximum SNR per resolution element for any input object of the FIREBall-2 IMO.

\subsection{Optimizing FIREBall observing strategy}

Now, we use our simulated haloes as input into the IMO and perform the above described SNR calculation for a $2 \mathrm{~h}$ exposure. From the results we then estimate the SNR of potential FIREBall targets.

For the SNR calculation, we choose the 10 most massive haloes at the redshifts for $\mathrm{C}$ IV, $\mathrm{Ly} \alpha$, and $\mathrm{O}$ VI $(0.3,0.7$, and 1.0 , respectively). The properties of these haloes are given in Table B1 in the appendix. We use the most massive haloes because they have the highest resolution in the AMR simulation. The most massive Ly $\alpha$ halo is shown in the upper two panels of Fig. 10.

We input those chosen 30 simulated galaxy haloes into the FIREBall IMO and determine their SNR map with the prescription given above. In the lower panel of Fig. 10, we show an example of the SNR map after data reduction for the most massive Ly $\alpha$ halo. From the SNR map, we determine the maximum SNR and plot it against the NUV AB magnitude of the stellar continuum of the input halo (scatter points in Fig. 11).

By relating the maximum SNR of the ELE to the NUV magnitude, we can compare the simulated haloes to actual galaxies and estimate which NUV magnitude corresponds to which maximum SNR given the emission line and redshift. Since we chose the most massive haloes from the simulation - which are supposedly also some of the brightest - we extrapolate from our results to lower magnitudes. From the extrapolation we determine how many galaxies have to be stacked to give a reasonable SNR.

We present our simple estimate in the following:

$\mathrm{SNR}=\frac{S}{N}=\frac{S}{\sqrt{S+B}}$.

Here, we assumed all background components to be inside $B$. Given the many uncertainties in determining the necessary parameters to calculate $B$, we simplify our analysis by considering the two extreme cases, each assumed to be valid in our complete magnitude range: One where $S \gg B$, which is optimistic (case 1), and another one where $S \ll B$, which is a pessimistic case (case 2 ). This gives us the following approximations for SNR:

$\operatorname{SNR}($ case 1$)=\frac{S}{\sqrt{S+B}} \approx \sqrt{S}$,

$\mathrm{SNR}($ case 2$)=\frac{S}{\sqrt{S+B}} \approx \frac{S}{\sqrt{B}} \propto S$.

We also assume the ELE flux to be proportional to the total galaxy flux and relate the magnitude/flux of the galaxy to signal from the 

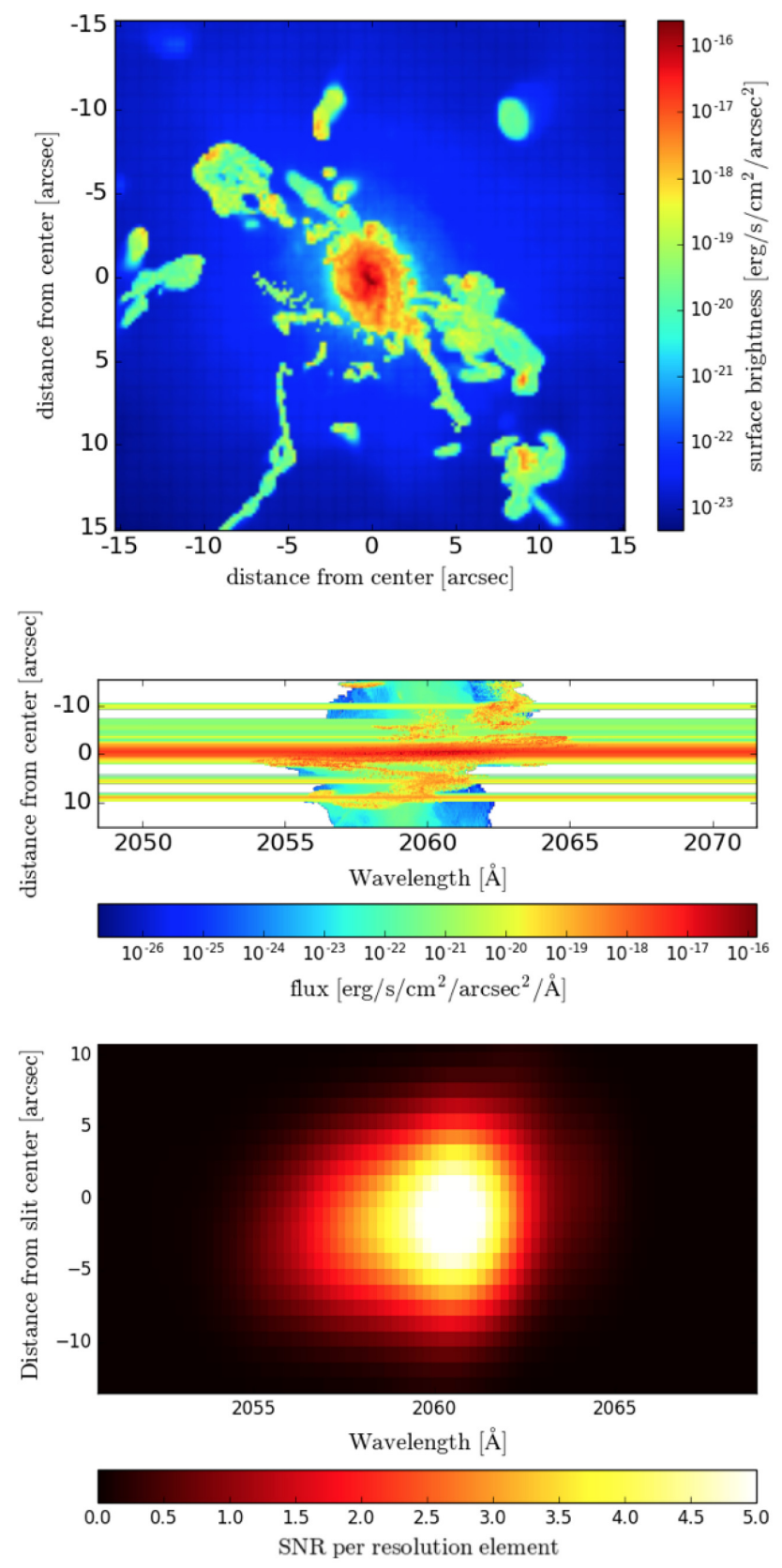

Figure 10. Example of the FIREBall IMO input and output: Here, we show the most massive Ly $\alpha$ halo input cube at $z=0.7$, illustrating the expected CGM flux from hydrodynamical RAMSES simulations, and output SNR. The upper panel shows the surface brightness of the simulated halo cube, the middle panel the projected spectrum of the same cube. The lower panel shows the output SNR per resolution element for this simulated galaxy halo, after going through the IMO and data reduction, including removal of the galaxy continuum. The output is for a $2 \mathrm{~h}$ observation.

ELE: $F \propto S$.

$m_{1}-m_{2}=-2.5 \log _{10}\left(\frac{F_{1}}{F_{2}}\right)=-2.5 \log _{10}\left(\frac{S_{1}}{S_{2}}\right)$.

Now we can relate the SNR for both cases to the difference in magnitudes. We assume B to be constant in case2. Given our results that the ELE from a galaxy with a continuum magnitude of 17.5 results in an SNR for Ly $\alpha$, we extrapolate to lower magnitudes with

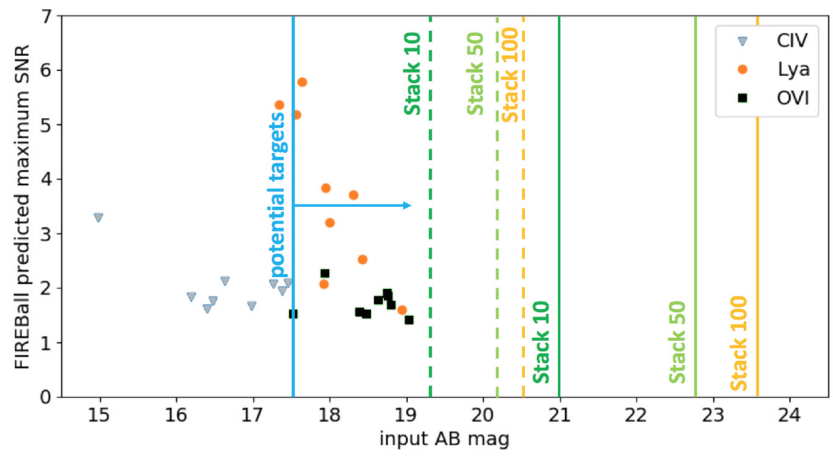

Figure 11. Expected SNR for FIREBall targets: The different markers show the calculated SNR for the 10 most massive haloes in the cosmological simulation at each redshift ( 0.3 for $\mathrm{C}$ IV, 0.7 for $\mathrm{Ly} \alpha$, and 1.0 for $\mathrm{O} \mathrm{VI}$ ). The blue line shows the limit of the brightest potential targets for FIREBall-2. The typical target for FIREBall-2 will, however, be fainter than this upper limit. We considered two cases: The optimistic case (solid lines), where the ELE signal dominates the background and a pessimistic case (dashed lines), where the background dominates the observations. The dark green lines shows the magnitude limit providing an SNR of 3 from stacking 10 Ly $\alpha$ sources in either case. The light green (yellow) lines shows the same limit from stacking 50 (100) sources.

the following expression:

$\operatorname{SNR}($ case 1$)=10^{\left(\frac{17.5-m}{5}\right)} \times 5$,

$\operatorname{SNR}($ case 2$)=10^{\left(\frac{17.5-m}{2.5}\right)} \times 5$.

A table with specific values for given input magnitudes is given in the appendix (Table B2). From these results we estimate that we will get good results for single galaxies at magnitudes up to NUV $\approx 18$, even in the pessimistic case. For fainter galaxies (NUV > 19) we will have to stack single observations to reach a good SNR (3) in either case. We assume to stack targets that give the same mean SNR individually and can thereby estimate the SNR that such a stack would give, at a given magnitude/SNR:

$\mathrm{SNR}_{\text {stack }}=\sqrt{\text { Number of Objects }} \times \mathrm{SNR}_{\text {individual object }}$.

Column 3 of Table B2 gives the number of targets that need to be stacked at a given magnitude to reach the desired SNR of 3 for both cases. In Fig. 11, we show our results as lines for the magnitude limits providing an SNR of 3 by stacking 10, 50, or 100 galaxies, where the dashed lines represent the pessimistic case and the solid lines the optimistic case. The ELE SNR for O VI and C IV is found to be low even for sources that are bright in UV continuum.

\subsection{Target selection}

Based on these findings, we optimized the target selection and observing strategy for the launch in 2018 September. We favour bright quasars and Ly $\alpha$ emitting galaxies over the metal lines and aim primarily at dense fields with groups of quasars and $\mathrm{Ly} \alpha$ galaxies in order to boost the signal through feedback.

The typical galaxy that qualifies as a target for FIREBall has a mean NUV magnitude of $\operatorname{mag}_{\mathrm{NUV}} \sim 23-24$. Therefore, we aim to observe as many targets as possible during the night, in order to perform a stacking analysis. In order to maximize the number of targets, we prepared four fields that should ideally be observed in equal amounts of time, resulting in $2 \mathrm{~h}$ per field for a $8 \mathrm{~h}$ 
night observation. From our SNR results we know that for a $2 \mathrm{~h}$ observation we can get a good SNR for the bright objects while the faint ones need to be stacked. Each target field consists of up to $\sim 80$ targets that fall into the right redshift windows. Wherever there was still space in the field (on the mask), we put also some metal line galaxies, to make the best use of the detector.

In addition to the four science fields that need to be prepared in advance for mask cutting, the instrument will be equipped with a single slit for more flexible observations of e.g. an additional bright quasar, since bright targets are the most promising for the FIREBall observations.

\subsection{Expectations from the FIREBall experiment}

We analysed the possible detection of CGM faint emission - or extended line emission ELE - from low-redshift galaxies with the FIREBall-2 UV MOS. We used mock cubes of an emission model on the FIREBall instrument model reproducing the output of the FIREBall-2 detector. The two-dimensional analysis of the signal indicates that the massive objects can be observed in Ly $\alpha$ at redshift $z=0.67$ within the time available for the balloon's flight. This shows the need for future development for the satellite version of the instrument, ISTOS. Our simulations indicate that with the current version of the instrument and flight-plan it will be challenging to detect the OVI and CIV emission lines (at redshift 1.0 and 0.3, respectively).

We also considered stacking in order to achieve observability and a good SNR for the ELE. For this we reviewed the continuum magnitudes of galaxies that can be potential targets for FIREBall2. The brightest FIREBall-2 targets have magnitudes NUV $\sim 18$. Those, including quasars, will give an excellent SNR with a single observation. For the fainter targets $(19<\mathrm{NUV}<21)$ we would need to stack 10-300 galaxies to reach a good SNR. Galaxies fainter than NUV $=21$ - including the bulk of the FIREBall-2 targets with a mean NUV $\sim 23-24-$ will be challenging to observe even when stacking the signal, when assuming that the observations are dominated by the background. In the other extreme case, where the observations are dominated by the signal of the object, we expect to obtain the desired SNR of 3 when stacking $\sim 100-300$ galaxies down to NUV $=24$. The real case will lie somewhere in-between those two extremes.

One remaining issue with the FIREBall-2 observations will be the separation of the CGM from the disc line emission. With the current spatial resolution we would need a highly luminous and extended CGM to resolve it separately from the disc. In case this is not possible, we will have to make assumptions on the ratio between Ly $\alpha$ disc emission and CGM emission and apply it to the total signal in order to estimate the CGM flux.

Future satellite missions like ISTOS (Martin 2014) or LUVOIR (France et al. 2017) will enable us to see the UV emission of galaxies at these redshifts at an even better SNR and thus will be able to spatially resolve the CGM.

\section{OPTICAL AND NEAR-INFRARED OBSERVATIONS WITH ELT/HARMONI}

The High Angular Resolution Monolithic Optical and Near-infrared Integral field spectrograph ${ }^{5}$ (HARMONI, PI: N.A. Thatte; Thatte et al. 2014) will be the integral field spectrograph (IFS) at the ESO

\footnotetext{
${ }^{5}$ http://www-astro.physics.ox.ac.uk/instr/HARMONI/
}

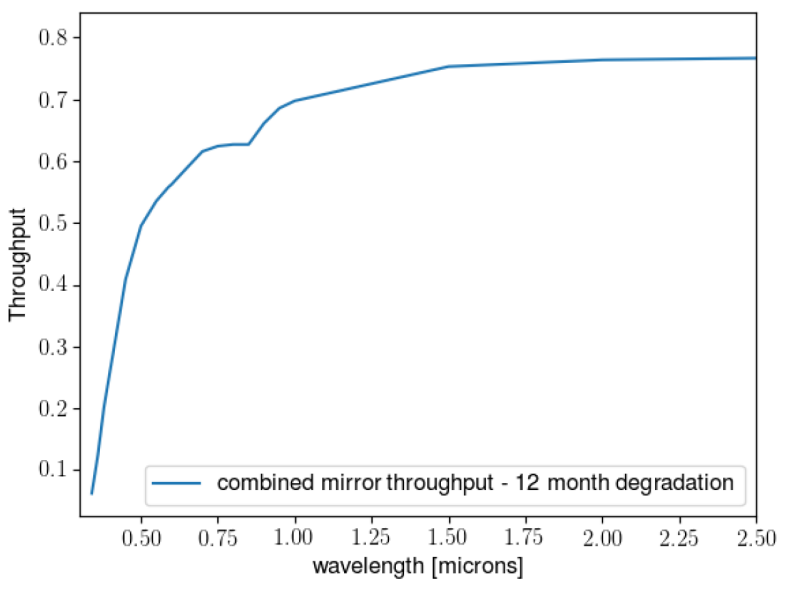

Figure 12. Combined reflectivity of the six mirrors in the ELT for a 12 month degradation. While the throughput is more than 70 per cent at NIR wavelengths the visible, and especially the blue wavelengths are heavily absorbed. Curve taken from HSIM (Zieleniewski et al. 2015) version 115. It assumes a Gemini 4 Layer Ag coating for five of the six mirrors in the ELT and a fresh Al coating for one of them (Hass 1965; Maxime Boccas 2004).

Extremely Large Telescope ${ }^{6}$ (ELT). HARMONI will be available with different flavors of adaptive optics (AO) systems. It can be used without AO, with Laser Tomography AO (LTAO) or with Single Conjugate AO. The instrument will cover a wavelength range from $0.47 \mu \mathrm{m}$ in the visible to $2.45 \mu \mathrm{m}$ in the near-infrared. There will be four different spatial scales available with associated fields of view. For our purposes we will only consider the widest field of view, which has the biggest spaxels. This coarser spatial resolution mode with spaxels of a size of $60 \times 30$ mas will have the largest field of view $(6.42 \times 9.12 \operatorname{arcsec})$, which is most appropriate to study the large extent of the CGM outside the host galaxies. HARMONI can achieve a spectral resolution between $R \sim 3000$ and $R \sim 20000$, depending on the wavelength regime. The ELT and HARMONI are planned to have first light in late 2024.

\subsection{The HARMONI instrument simulator}

In preparation of the science objectives with HARMONI, a simulation tool called HSIM (HARMONI instrument simulator) ${ }^{7}$ has been developed (Zieleniewski et al. 2015). It is an instrument model and calculates the observed signal and noise for a given input source, taking into account all instrumental and atmospheric effects. In particular, it takes into account the reflectivity of the telescope mirror coating and its degradation over time. This is particularly essential for our science objective, as the coating shows poor performance in the blue. At $5000 \AA$ an overall reflectivity of $\sim 50$ per cent or less is expected (for reflections on the six mirrors of the telescope, see Fig. 12), depending on the state of degradation. HSIM returns a reduced mock observation of the input object. We use version 115 of HSIM to determine the expected signal from the CGM using HARMONI.

We prepare three-dimensional data cubes of simulated galaxy haloes in a similar way as for the FIREBall IMO. For the observation simulations we use the $V+R, I z+J$, and $H+K$ gratings, giving a spectral resolution of $R=3100-3300$ and the coarse spaxel scale

\footnotetext{
${ }^{6}$ https://www.eso.org/sci/facilities/eelt/

${ }^{7}$ https://github.com/HARMONI-ELT/HSIM
} 
Table 6. Redshift coverage of different lines with HARMONI (0.47-2.45 $\mu \mathrm{m})$.

\begin{tabular}{lcc}
\hline Line & Redshift range & Chosen redshift for simulations \\
\hline H $\alpha$ & $0.0-2.7$ & $0.3,1,2$ \\
Ly $\alpha$ & $2.9-19$ & $4,6,10$ \\
O VI & $3.5-22$ & 4 \\
C IV & $2.0-14$ & 3 \\
\hline
\end{tabular}

with pixels of $30 \times 60$ mas. For AO we use the LTAO that uses laser guide stars. Given our setup (biggest spaxels), a tip-tilt star free mode will provide a full sky coverage. We set the Zenith seeing to 0.67 arcsec and the Zenith angle to $0 \mathrm{deg}$. The telescope temperature is set to $280.5 \mathrm{~K}$ as the default temperature given by HSIM.

\subsection{Simulated input cubes}

As for FIREBall, we use the post-processed galaxy halo simulations described earlier. We consider $\mathrm{Ly} \alpha, \mathrm{O}$ VI and $\mathrm{C}$ IV as potential tracers for CGM emission. Additionally, we use $\mathrm{H} \alpha$ as a tracer for lowredshift CGM. Table 6 gives an overview of the lines and their respective redshifts. At each redshift we consider the most massive halo, because they have the highest resolution in the AMR RAMSES simulation. These haloes are investigated for the general CGM properties such as angular extent and luminosities (see Section 6.3).

For the HSIM input and the estimation of flux-dependent SNR at different wavelengths we use the most massive halo at redshift 0.3 . The properties of this halo are given in the first line in Table B1. The line we choose in this halo is $\mathrm{H} \alpha$. We pick this halo because of its high resolution and gas-rich CGM. Defining an area of $0.6 \times 0.6$ $\operatorname{arcsec}^{2}$ around a gas cloud in its CGM, we want to know how the SNR of the flux in this area changes with wavelength. Therefore, we shift the input cube's wavelength in steps of $\Delta \lambda=0.05 \mu \mathrm{m}$ to populate the spectral coverage of HARMONI. For each of these cubes at different wavelengths, we also modify the flux by scaling by factors ranging from $10^{-4}$ to $10^{4}$ in $9 \log$ steps. Thereby we end up with 9 different input fluxes at each wavelength for which we measure the output SNRs.

In Fig. 13, we show one example of input and output of HSIM. The upper two panels show the $\mathrm{H} \alpha$ surface brightness map of the most massive halo at redshift 0.3 . This halo was modified according to the above description and shifted in wavelength, so that the lower panel shows the SNR for the cube at a wavelength of $1.32 \mu \mathrm{m}$.

\subsection{CGM evolution and observability}

The extended wavelength range of HARMONI will allow us to observe different CGM tracers at various redshifts (see Table 6). We know that at low redshifts, the CGM can reach out to several hundreds of kpc (Tumlinson et al. 2017). The maximum field of view of HARMONI is $9 \times 6 \operatorname{arcsec}^{2}$. At $z=0.3,1 \operatorname{arcsec}$ corresponds to $\sim 4.5 \mathrm{kpc}$. It will not be possible to map the full CGM region in one exposure with HARMONI at this redshift. Due to cosmic evolution, angular scale will be smallest between $z=1$ and $z=2$. Beyond $z \sim 1-2$, the CGM will appear even smaller due to the early stages of galaxy evolution itself but also fainter due to redshift effects. Therefore, observations of galaxy haloes at $z \sim$ 1-2 will be optimal to map the CGM. At these redshifts the largest field of view of HARMONI of $9 \times 6$ arcsec will correspond to $\sim 75 \times 50 \mathrm{kpc}$. The virial radii of galaxy haloes at those redshifts can stretch out to $\sim 200-300 \mathrm{kpc}$, so the majority of the surroundings
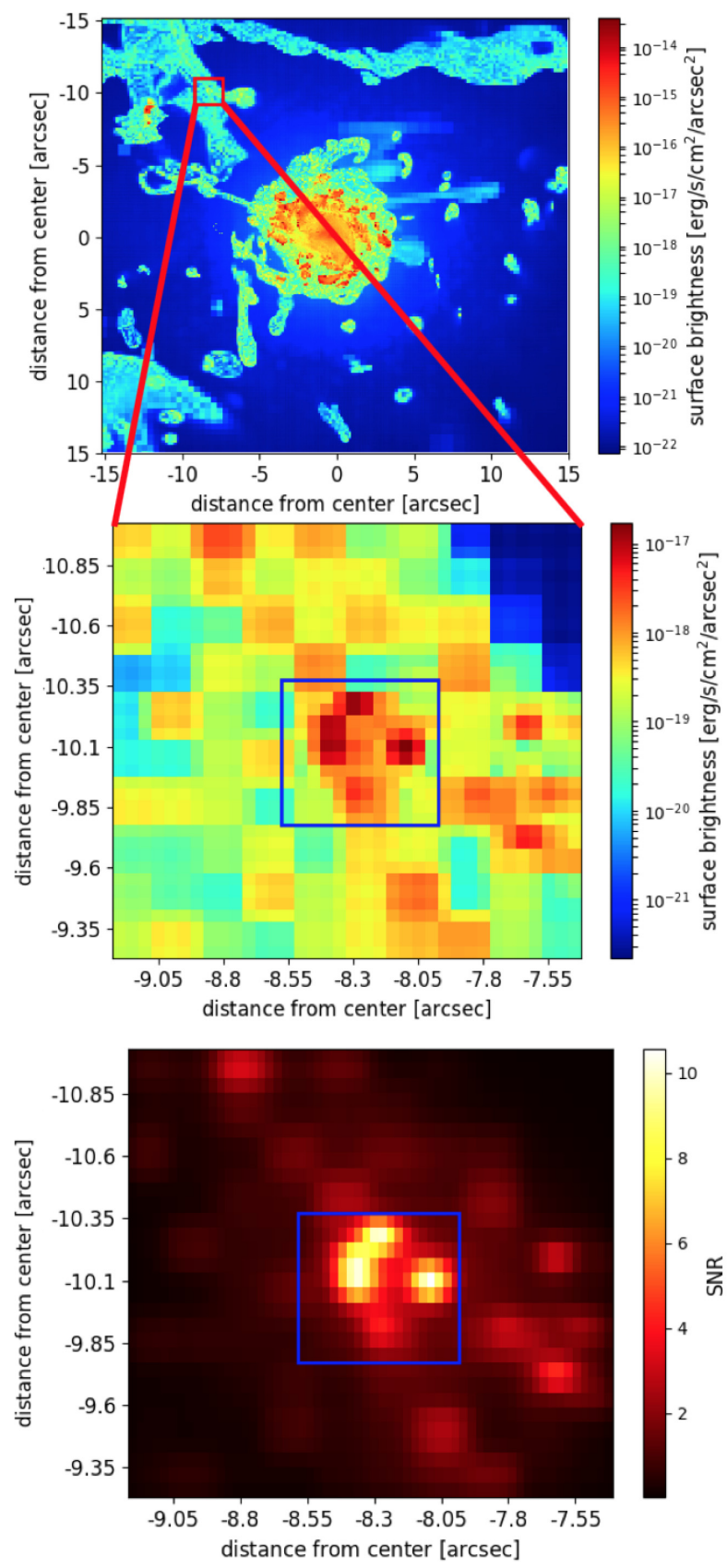

Figure 13. Example of HSIM input and output: The upper panel shows the $\mathrm{H} \alpha$ emission in the most massive galaxy halo at redshift 0.3 in our RAMSES AMR hydrodynamical simulations. From this cube - in order to save computation time - we extract a smaller data cube from one of the filaments in the CGM region around the central galaxy (middle panel). The cube was shifted to different wavelengths in order to populate the spectral range of HARMONI. In the bottom panel we show the output SNR for the input cube at $1.32 \mu \mathrm{m}$ in the $I z+J$ grating for an integration time of $5 \mathrm{~h}$. The blue box in the lower two panels gives the region of $0.6 \operatorname{arcsec} \times 0.6 \operatorname{arcsec}$, which is considered for the analysis.

of a galaxy could be covered with $\sim 4$ neighbouring exposures. For Ly $\alpha$ that is the brightest line at any redshift, we conclude that the optimal redshift for CGM observations is $z \gtrsim 3$, because from redshift 3 the virial radii of galaxies will typically be $<70 \mathrm{kpc}$ and it will be possible to capture the CGM in a single exposure. 

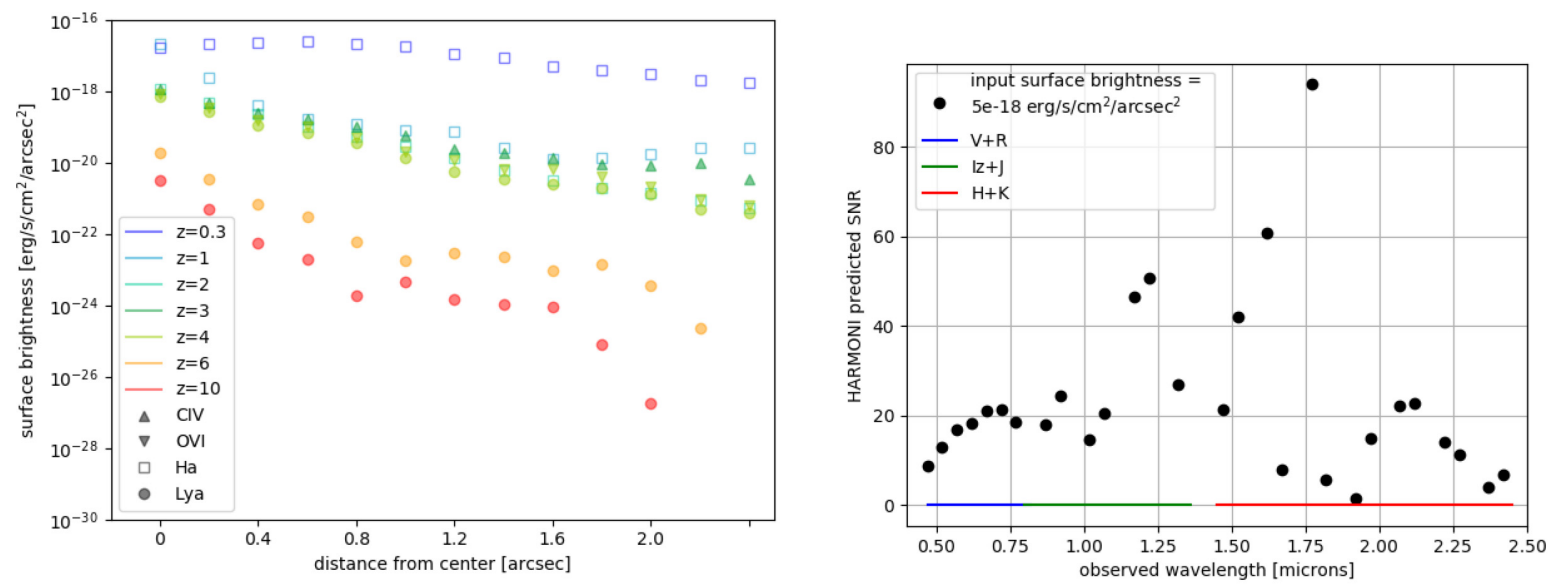

Figure 14. Left-hand panel: Radial profiles of simulated galaxy haloes: We show the radial surface brightness profiles of galaxies from our cosmological RAMSES AMR simulations for different ions at various redshifts. On the $x$-axis we plot the distance from the centre of the galaxy halo in units of arcsec, which are independent of redshift and therefore translate to different physical sizes for a given redshift. The points give the average surface brightness (on $y$-axis) of the halo at a given distance. As expected, the overall surface brightness decreases with redshift. We also notice that Ly $\alpha$ emission at redshift 4 is comparable to $\mathrm{H} \alpha$ emission at $z=2$ and metal lines at redshifts 3 and 4. Right-hand panel: Wavelength dependence of output SNR in HARMONI: All SNR points are calculated for a given input surface brightness of $5 \mathrm{e}-18 \mathrm{erg} \mathrm{s}^{-1} \mathrm{~cm}^{-2} \operatorname{arcsec}^{-2}$. The ranges of the $V+R, I z+J$, and $H+K$ gratings are shown in blue, green, and red, respectively. We see a steady increase of SNR in the blue that traces the throughput of the telescope, given a coating on the mirror that absorbs heavily at these wavelengths. The throughput in the NIR is higher and more steady than in the visible, but subject to atmospheric absorption lines and OH emission lines (especially in the $H+K$ band) so that the SNR varies strongly with the precise wavelength.

To illustrate the flux evolution within a given angular size of 2 arcsec - corresponding to $\sim 9-17 \mathrm{kpc}$, depending on the redshift we plotted in Fig. 14 the radial profiles of the most massive haloes at the redshifts and for the lines given in Table 6. Naturally, the low-redshift halo at $z=0.3$ gives the brightest flux profile. We also see the steep drop in luminosity for Ly $\alpha$ between $z=4,6$ and 10. Ly $\alpha$, being the brightest emission line in any galaxy halo, also shows comparable fluxes at $z=4$ to $\mathrm{H} \alpha$ emission at $z=2$ and metal lines at redshifts 3 and 4 .

\subsection{Predicted signal from HARMONI observations}

We run HSIM for a grid of wavelengths and fluxes as described in Section 6.2. The observing time is chosen to be $5 \mathrm{~h}$ with five integrations of $3600 \mathrm{~s}$ each. To optimize the observation setup, we tested different exposure settings in the $V+R$ grating and found that longer integration times and fewer exposures give a better SNR than choosing more exposures with shorter integration times. Specifically for the fixed $5 \mathrm{~h}$, we find a 7 percent increase of the SNR when choosing $5 \times 3600$ s over $20 \times 900$ s.

After running HSIM for each of our input cubes, we determine the corresponding SNR of the output. In the input cubes we have chosen an area of $0.6 \times 0.6 \mathrm{arcsec}^{2}$ around a gas clump. In the output cubes, we derive the SNR of the same area by binning $20 \times 10$ pixels in the HSIM output.

First, we consider the input cubes with the original input flux from the cosmological simulations. We investigate the wavelength dependence of the output SNR for a given flux (Fig. 14). While the SNR increases with wavelength in the visible, just as expected from the telescope's throughput, there is a large scatter of SNRs at larger wavelengths. At these wavelengths the instrument's throughput is better than in the visible, resulting in high SNRs. But there are also numerous atmospheric absorption lines and $\mathrm{OH}$ emission lines that corrupt the observation and lead to low SNRs. By choosing random input wavelengths, some regions are affected by the atmosphere and some others are not (e.g. $1.77 \mu \mathrm{m})$

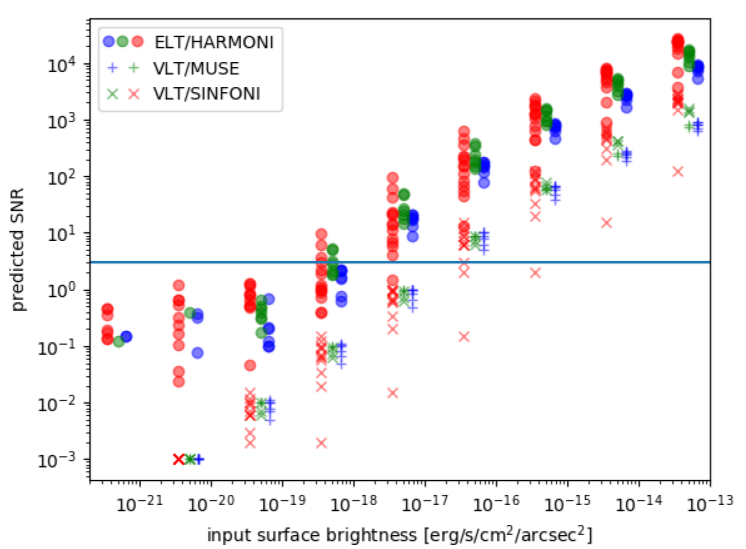

Figure 15. Expected signal to noise for different input fluxes and comparison to existing IFSs (MUSE and SINFONI) for a $5 \mathrm{~h}$ observation. We plot for each pair of surface brightness and wavelength the output SNR. The circles give the SNR for HARMONI, the ' + ' the SNR for MUSE and the ' $x$ ' the SNR for SINFONI. We group wavelengths that would fall into the $V+R, I z+J$, and $H+K$ band of HARMONI with blue, green, and red colours, respectively. For display purposes, we offset the points in the different wavelength bands in $x$-direction. The green points are at the original surface brightness, the red and blue ones have been shifted to the left and right, respectively. We also show the SNR $=3$ limit, which is the minimum SNR that should be achieved for kinematic modelling (Bouché et al. 2015; Péroux et al. 2017). We find a significant increase of SNR in HARMONI compared to both MUSE and SINFONI. The ratios between HARMONI and MUSE SNR are shown in Fig. 16, the ratios between HARMONI and SINFONI SNR are shown in Fig. 16.

and give an indication of the range of SNR in HARMONI NIR observations.

We also use the flux-modified input cubes at each wavelength and determine how the SNR changes with both input flux and wavelength. In Fig. 15, we show the result of this computation. We plot the output SNR against input flux. The colour-coding 

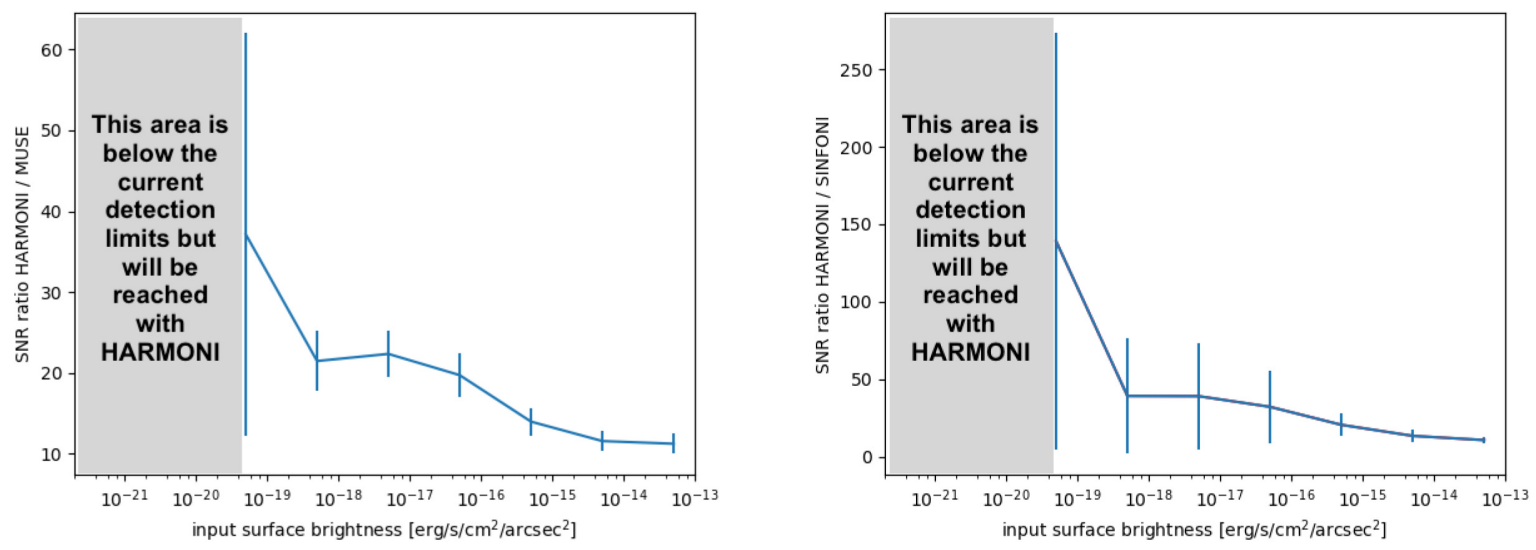

Figure 16. Left-hand panel: SNR ratio between HARMONI and MUSE: For each wavelength and input flux, we determine the ratio between SNR in HARMONI and SNR in MUSE. For each value in input flux we compute the mean and standard deviation of these ratios at all wavelengths. We find an increase of SNR in HARMONI over MUSE at all wavelengths and fluxes of at least an order of magnitude. We predict a steepening increase of this ratio towards low surface brightness. The standard deviation increases significantly at low surface brightness as the SNR in the MUSE data becomes very low and a large spread of SNR ratios becomes possible. This means that faint diffuse emission from the CGM that is currently not detectable in even the deepest MUSE observations will become observable in HARMONI. Right-hand panel: same as left-hand panel but for SINFONI.

corresponds to the chosen grating. For each flux there is a spread in SNRs for each grating because of the different wavelengths we analysed within each grating.

\subsection{Comparison with current IFSs at optical/NIR wavelengths}

To quantify the gain from ELT/HARMONI over current state-ofthe-art IFSs such as MUSE and SINFONI on the VLT, we compare our findings for the expected SNR of the CGM to the SNR we expect to have with these instruments for the same given flux of the CGM. To do so we use the online exposure time calculators (ETCs) for both $\mathrm{MUSE}^{8}$ and SINFONI. ${ }^{9}$

\subsubsection{Optical IFS VLT/MUSE}

MUSE is an IFS at the VLT and covers a wavelength range from 480 to $930 \mathrm{~nm}$. It has been successfully used in tracing extended Ly $\alpha$ emission around galaxies (e.g. Wisotzki et al. 2016). We use version P102.7 of the MUSE ETC to calculate the expected SNR of the same line emission as for HARMONI with HSIM. For the source emission we assume an extended source with 1.13 arcsec diameter (to result in $1 \operatorname{arcsec}^{2}$ area) and single line emission at the same wavelengths and fluxes as for HARMONI. We use the Wide Field Mode without $\mathrm{AO}$ and a spatial binning of $3 \times 3$ pixels to reach the same area of $0.6 \times 0.6 \operatorname{arcsec}^{2}$ as in the simulation. We assume an airmass of 1.5 , moon FLI of 0.5 and seeing of 0.67 arcsec. The exposure time is also set to the same amount as for HSIM: $5 \times 3600$ s. Our results for the obtained SNR in MUSE is plotted in Fig. 15 with crosses $(+)$ and in colours blue and green, corresponding to the wavelength ranges of the HARMONI gratings.

We find an overall increase of a factor $\sim 20$ in SNR for HARMONI observations over MUSE observations. The ratios of SNRs (HARMONI/MUSE) are shown in Fig. 16. While there is generally an increase of more than one order of magnitude at

\footnotetext{
${ }^{8} \mathrm{http} / / /$ www.eso.org/observing/etc/bin/gen/form?INS.MODE=swspectr+I NS.NAME $=$ MUSE

${ }^{9}$ https://www.eso.org/observing/etc/bin/gen/form?INS.NAME=SINFON I+INS.MODE $=$ swspectr
}

fluxes $>10^{-18} \mathrm{erg} \mathrm{s}^{-1} \mathrm{~cm}^{-2} \operatorname{arcsec}^{-2}$, we also find that small fluxes that would have been undetected even in deep observations with MUSE (detection limit for emission lines in $\sim 20-30 \mathrm{~h}$ MUSE observations is $(2.8-5.5) \times 10^{-20} \mathrm{erg} \mathrm{s}^{-1} \mathrm{~cm}^{-2} \operatorname{arcsec}^{-2} \AA^{-1}$ in the most ideal cases (Leclercq et al. 2017) generally it is around $\sim 1 \times 10^{-19} \mathrm{erg} \mathrm{s}^{-1} \mathrm{~cm}^{-2} \operatorname{arcsec}^{-2}$, Wisotzki et al. 2016; Bacon et al. 2017; Wisotzki et al. 2018) will become observable in HARMONI. Thus, HARMONI will enable new CGM science. This will mark the next step in CGM studies, where we are limited by the current instrument sensitivities. We will be able to map the CGM and get measurements on its extent and clumpiness. Notwithstanding the ELT's mirror coatings, which is suboptimal at visible wavelengths, the increase in collecting area means that photon-starved science cases would benefit from the ELT even at visible wavelengths.

\subsubsection{NIR IFS VLT/SINFONI}

SINFONI, is the NIR IFS at the VLT and operating in the nearinfrared from 1.1 to $2.45 \mu \mathrm{m}$. We vary the input flux and wavelength and determine the output SNR with the SINFONI ETC version P102.7. We assume an extended source, with an area of $0.36 \operatorname{arcsec}^{2}$, because the output SNR in the SINFONI ETC is given for the entire source size. The AO and sky conditions are the same as for the MUSE ETC. The angular resolution scale is set to 250 milliarcsec to get the maximum sensitivity and biggest FOV and we use the $J$-, $H$ - and $K$-band grating for the respective wavelengths. We assume a total exposure time of $5 \mathrm{~h}$ but due to the sky variations at NIR wavelengths in SINFONI we split it into $20 \times 900$ s. Our results are again plotted in Fig. 15 with an ' $x$ ' and in red and green, corresponding to the respective gratings in HARMONI.

We find an increase of at least a factor $\sim 15$ for HARMONI observations over SINFONI observations, with a mean between a factor 15-100. We plotted the ratios for all fluxes in Fig. 16: The expected SNR increases at all redshifts and the small fluxes that were previously not observable will become detectable with HARMONI. SINFONI will be decommissioned in $2019^{10}$ and replaced in 2020

\footnotetext{
${ }^{10} \mathrm{https}: / /$ www.eso.org/sci/facilities/paranal/cfp/cfp102/foreseen-changes.h
} tml 
by ERIS. ${ }^{11}$ By the mid-2020s, when the ELT will be available, HARMONI will make a more than suitable replacement for the only NIR IFS at large ESO telescopes.

\subsection{Future CGM studies with HARMONI}

As we have shown in Fig. 15, we expect HARMONI to detect at least one order of magnitude smaller fluxes than previously possible and we will be able to detect diffuse emission that is an order of magnitude fainter in surface brightness than the faintest detectable emissions discovered by MUSE and SINFONI. This means that ELT/HARMONI will be well suited for photon starved science cases such as the faint diffuse emission from the CGM. Even though the mirror coating of the ELT has suboptimal reflectivity at visible wavelengths, the telescope's large collecting area provides an improved signal with respect to VLT/MUSE observations.

\section{CONCLUSION}

We have dealt with the complex question of CGM faint emission modelling in order to produce realistic data cubes that can be used for observability predictions of the CGM with upcoming instruments. We have used a state-of-the-art high-resolution hydrodynamical cosmological RAMSES simulation to extract different massive haloes $\left(10^{13} \mathrm{M}_{\odot}\right)$. Using a photoionization code, we modelled different line emissivities considering the UVB fluorescence and the gravitational cooling of the gas. We also considered the stellar contribution to the gas fluorescence in the case of Ly $\alpha$ photons and we derived the level of the UV continuum in those wavelengths after attenuation by the ISM dust. Our simulations include feedback from supernova explosions, modelled such that it creates artificially hot 'delayed-cooling' cells. In our model we exclude those cells in order to stay conservative in terms of total luminosity.

We find our simulations to be in good agreement with low-redshift observations from GALEX (Deharveng et al. 2008; Wold et al. 2014, 2017) for Ly $\alpha$ escape fractions between 0.1 per cent and 1 per cent. Moving to higher redshifts ( $z=4.0$ and $z=2.33$ ), our CGM Ly $\alpha$ emission model agrees well with the observational data provided we use a lower Ly $\alpha$ escape fraction than is usually inferred from observations. This effect might originate from the stacking of a large number of objects in the $z=2.33$ case. Using our simulations, we can create simulated data cubes of mock observations with two spatial axes and one spectral axis.

We have also investigated the expected signals from CGM emission with two upcoming instruments: FIREBall-2 and HARMONI on the ELT. We used the simulated haloes as input into the respective instrument models of FIREBall-2 and HARMONI. From these simulations we get an estimate of the signal that faint diffuse emission gives in observations with each of these instruments. Those results give the base for target selection and observing strategies.

Our simulations and analysis have given us a basis on which targets to select - focusing on Ly $\alpha$ rather than the metal lines C IV and OVI. While observations of individual objects will be challenging and probably only bright UV objects like quasars provide a high SNR, the instrument is designed such that it will be able to observe several hundreds of galaxies in one night. Stacking the signal of several hundred galaxies will be the way of analysing the FIREBall-2 data to gain new insights into extended Ly $\alpha$ emission at low redshifts. FIREBall-2 was launched in September 2018 and

\footnotetext{
${ }^{11}$ https://www.eso.org/sci/facilities/develop/instruments/eris.html
}

observed the low $z$ CGM for the first time. The data analysis of FIREBall-2 data is currently ongoing.

HARMONI, which has successfully passed the Preliminary Design Review, is planned for first light in late 2024. The instrument design allows for a reliable instrument model that we use to prepare future CGM observations. HARMONI will be a visible and NIR IFS and able to target different CGM tracers at various redshifts. We have investigated the SNR expected for various input fluxes at different wavelengths and compared to the existing IFSs MUSE and SINFONI on the VLT. We find an increase of $\sim 20$ times better SNR with HARMONI compared to the current instruments. This will allow us to reach one order of magnitude fainter surface brightness of faint diffuse emission than current facilities and will enable CGM studies. Going to higher redshifts $(z \sim 1-2)$ will allow us to map larger areas and in combination with the less evolved galaxies and surroundings, it will be possible to map galaxies with their entire CGM. Therefore, we conclude on a 'sweet spot' at redshift $\sim 1-2$ for general CGM observations and $\operatorname{Ly} \alpha$ to be well observable at $z=3-4$. HARMONI will enter a regime of low surface brightness that is not attainable with current facilities. Also, while MUSE has a bigger field of view than HARMONI and is able to detect more galaxies in one exposure, HARMONI will allow us to reach lower surface brightness ( $\mathrm{SB}>\sim 10^{-19}-10^{-20} \mathrm{erg} \mathrm{s}^{-1} \mathrm{~cm}^{-2} \operatorname{arcsec}^{-2}$ ) in a 5 h exposure.

Overall, the future looks promising for CGM studies with many upcoming new instruments, such as ISTOS (Martin 2014) or LUVOIR Ultraviolet Multi-Object Spectrograph (LUMOS; France et al. 2017). Apart from the instruments that we have studied in this work it will be important to assess how space-based X-ray missions like ATHENA will shed new light on to the hot gas content of galaxy haloes and address the missing baryon problem in the low-redshift universe (Nicastro et al. 2018).

\section{ACKNOWLEDGEMENTS}

RA thanks CNRS and CNES (Centre National d'Etudes Spatiales) as well as ESO for support for her $\mathrm{PhD}$. CP is grateful to the ESO science visitor programme and the DFG cluster of excellence 'Origin and Structure of the Universe' for support. CP thanks the Alexander von Humboldt Foundation for the granting of a Bessel Research Award held at MPA. NT, LR, MPS, and SZ acknowledge support from the Science and Technology Facilities Council (grants ST/N002717/1 and ST/M007650/1), as part of the UK E-ELT Programme at the University of Oxford. The simulations in this work are part of the BINGO! project. This work was granted access to the HPC resources of CINES under the allocation 2015042287 made by GENCI. Images created with GLNemo2 (https: //projets.lam.fr/projects/glnemo2), a 3D visualization program for nbody data, developed by Jean-Charles Lambert at CeSAM-LAM. We thank J.-C. Lambert for GLNemo2-related discussions. We thank ESO for the publicly available Exposure Time Calculators for MUSE and SINFONI. RA thanks Jérémy Fensch and Nicolas Guillard for useful discussions.

\section{REFERENCES}

Agertz O., Teyssier R., Moore B., 2011, MNRAS, 410, 1391

Arrigoni Battaia F., Hennawi J. F., Prochaska J. X., Cantalupo S., 2015, ApJ, 809,163

Arrigoni Battaia F., Hennawi J. F., Cantalupo S., Prochaska J. X., 2016, ApJ, 829,3 
Arrigoni Battaia F., Prochaska J. X., Hennawi J. F., Obreja A., Buck T., Cantalupo S., Dutton A. A., Macciò A. V., 2018, MNRAS, 473, 3907

Arrigoni Battaia F., Hennawi J. F., Prochaska J. X., Oñorbe J., Farina E. P., Cantalupo S., Lusso E., 2019, MNRAS, 482, 3162

Augustin R. et al., 2018, MNRAS, 478, 3120

Bacon R. et al., 2014, The Messenger, 157, 13

Bacon R. et al., 2017, A\&A, 608, A1

Bertone S., Schaye J., Booth C. M., Dalla Vecchia C., Theuns T., Wiersma R. P. C., 2010, MNRAS, 408, 1120

Bertone S., Schaye J., 2012, MNRAS, 419, 780

Boccas M., Vucina T., Araya C., Vera E., Ahhee C., 2004, in AtadEttedgui E., Dierickx P., eds, Proc. SPIE Conf. Ser. Vol. 5494, Optical Fabrication, Metrology, and Material Advancements for Telescopes. SPIE, Bellingham, p. 239

Borisova E. et al., 2016, ApJ, 831, 39

Bottorff M. C., Ferland G. J., Straley J. P., 2006, PASP, 118, 1176

Bouché N., Carfantan H., Schroetter I., Michel-Dansac L., Contini T., 2015, AJ, 150, 92

Bournaud F., Dekel A., Teyssier R., Cacciato M., Daddi E., Juneau S., Shankar F., 2011, ApJ, 741, L33

Calzetti D., Armus L., Bohlin R. C., Kinney A. L., Koornneef J., StorchiBergmann T., 2000, ApJ, 533, 682

Cantalupo S., 2017, in Fox A., Davé R., eds, Astrophysics and Space Science Library, Vol. 430, Gas Accretion on to Galaxies. Springer, Berlin, p. 195

Cantalupo S., Porciani C., Lilly S. J., Miniati F., 2005, ApJ, 628, 61

Cantalupo S., Arrigoni-Battaia F., Prochaska J. X., Hennawi J. F., Madau P., 2014, Nature, 506, 63

Ceverino D., Klypin A., 2009, ApJ, 695, 292

Corlies L., Peeples M. S., Tumlinson J., O’Shea B. W., Lehner N., Howk J. C., O’Meara J. M., 2018, preprint (arXiv:e-print)

Costa T., Sijacki D., Haehnelt M. G., 2014, MNRAS, 444, 2355

Cox D. P., 2005, ARA\&A, 43, 337

Deharveng J.-M. et al., 2008, ApJ, 680, 1072

Dekel A. et al., 2009, Nature, 457, 451

Dijkstra M., Jeeson-Daniel A., 2013, MNRAS, 435, 3333

Dijkstra M., Loeb A., 2009, MNRAS, 400, 1109

Fardal M. A., Katz N., Gardner J. P., Hernquist L., Weinberg D. H., Davé R., 2001, ApJ, 562, 605

Faucher-Giguère C.-A., Kereš D., Dijkstra M., Hernquist L., Zaldarriaga M., 2010, ApJ, 725, 633

Ferland G. J., Korista K. T., Verner D. A., Ferguson J. W., Kingdon J. B., Verner E. M., 1998, PASP, 110, 761

Fox A., Davé R., eds, 2017, Astrophysics and Space Science Library, Vol. 430, Gas Accretion on to Galaxies . Springer, Berlin

France K., et al., 2017, in Oswald H. S., ed., Proc. SPIE Conf. Ser. Vol. 10397, UV, X-Ray, and Gamma-Ray Space Instrumentation for Astronomy XX. SPIE, Bellingham, p. 1039713

Frank S. et al., 2012, MNRAS, 420, 1731

Furlanetto S. R., Schaye J., Springel V., Hernquist L., 2004, ApJ, 606, 221

Furlanetto S. R., Schaye J., Springel V., Hernquist L., 2005, ApJ, 622, 7

Governato F. et al., 2010, Nature, 463, 203

Grevesse N., Asplund M., Sauval A. J., Scott P., 2010, Ap\&SS, 328, 179

Gronke M., Bird S., 2017, ApJ, 835, 207

Haardt F., Madau P., 2001, in Neumann D. M., Tran J. T. V., eds, Clusters of Galaxies and the High Redshift Universe Observed in X-rays. XXXVIth Rencontres de Moriont, XXIst Moriont Astrophysics Meeting, March 10-17, Savoie, France

Haardt F., Madau P., 2012, ApJ, 746, 125

Hahn O., Abel T., Kaehler R., 2013, MNRAS, 434, 1171

Hass G., 1965, in Kingslake R., ed., Applied Optics and Optical Engineering, Volume III. Academic Press, Inc., New York, p. 309

Hayes M., Schaerer D., Östlin G., Mas-Hesse J. M., Atek H., Kunth D., 2011, ApJ, 730, 8

Hayes M., Melinder J., Östlin G., Scarlata C., Lehnert M. D., MannerströmJansson G., 2016, ApJ, 828, 49

Hopkins P. F., et al., 2019, preprint (arXiv:1905.04321)

Hummels C. B., Smith B. D., Silvia D. W., 2017, ApJ, 847, 59

Hummels C. B. et al., 2018, preprint (arXiv:e-print)
Katz N., Weinberg D. H., Hernquist L., Miralda-Escude J., 1996, ApJ, 457, L57

Kennicutt R. C., Jr., 1998, ARA\&A, 36, 189

Kereš D., Katz N., Weinberg D. H., Davé R., 2005, MNRAS, 363, 2

Khaire V., Srianand R., 2019, MNRAS, 484, 4174

Kollmeier J. A., Zheng Z., Davé R., Gould A., Katz N., Miralda-Escudé J., Weinberg D. H., 2010, ApJ, 708, 1048

Kollmeier J. A. et al., 2014, ApJ, 789, L32

Krogager J.-K., Møller P., Fynbo J. P. U., Noterdaeme P., 2017, MNRAS, 469,2959

Kunth D., Leitherer C., Mas-Hesse J. M., Östlin G., Petrosian A., 2003, ApJ, 597, 263

Lake E., Zheng Z., Cen R., Sadoun R., Momose R., Ouchi M., 2015, ApJ, 806,46

Leclercq F. et al., 2017, A\&A, 608, A8

Lokhorst D., Abraham R., van Dokkum P., Wijers N., Schaye J., 2019, ApJ, 877,4

Madau P., Dickinson M., 2014, ARA\&A, 52, 415

Martin C., 2014, in Detecting and Mapping Hidden Baryons in the Intergalactic Medium in the Ultraviolet. 40th COSPAR Scientific Assembly, Moscow, Russia

Martin D. C., Chang D., Matuszewski M., Morrissey P., Rahman S., Moore A., Steidel C. C., 2014, ApJ, 786, 106

Matthee J., Sobral D., Oteo I., Best P., Smail I., Röttgering H., PaulinoAfonso A., 2016, MNRAS, 458, 449

Mège P., Pascal S., Quiret S., Corlies L., Vibert D., Grange R., Milliard B., 2015, in Oswald H. S., ed., Proc. SPIE Conf. Ser. Vol. 9601, UV, X-Ray, and Gamma-Ray Space Instrumentation for Astronomy XIX. SPIE, Bellingham, p. 960110

Milliard B. et al., 2010, in Arnaud M., Murray S. S., Takahashi T., eds, Proc. SPIE Conf. Ser. Vol. 7732, Space Telescopes and Instrumentation 2010: Ultraviolet to Gamma Ray. SPIE, Bellingham, p. 773205

Momose R. et al., 2014, MNRAS, 442, 110

Naidu R. P. et al., 2017, ApJ, 847, 12

Nicastro F. et al., 2018, Nature, 558, 406

Noterdaeme P. et al., 2012, A\&A, 547, L1

Oppenheimer B. D. et al., 2016, MNRAS, 460, 2157

Oppenheimer B. D., Schaye J., Crain R. A., Werk J. K., Richings A. J., 2018, MNRAS, 481, 835

Peeples M. S. et al., 2019, ApJ, 873, 129

Pengelly R. M., Seaton M. J., 1964, MNRAS, 127, 165

Péroux C. et al., 2017, MNRAS, 464, 2053

Pettini M., Shapley A. E., Steidel C. C., Cuby J.-G., Dickinson M., Moorwood A. F. M., Adelberger K. L., Giavalisco M., 2001, ApJ, 554, 981

Picouet V., et al., 2018, in den Herder J.-W.A., Nikzad S., Nakazawa K., eds, Proc. SPIE Conf. Ser., Space Telescopes and Instrumentation 2018: Ultraviolet to Gamma Ray. SPIE, Bellingham, p. 10699

Pieri M. M. et al., 2014, MNRAS, 441, 1718

Popping A., Davé R., Braun R., Oppenheimer B. D., 2009, A\&A, 504, 15

Quiret S. et al., 2016, MNRAS, 458, 4074

Rahmani H. et al., 2016, MNRAS, 463, 980

Rauch M. et al., 2008, ApJ, 681, 856

Rosdahl J., Blaizot J., 2012, MNRAS, 423, 344

Rosdahl J., Blaizot J., Aubert D., Stranex T., Teyssier R., 2013, MNRAS, 436, 2188

Salem M., Bryan G. L., Corlies L., 2016, MNRAS, 456, 582

Schaye J., 2001, ApJ, 562, L95

Schaye J. et al., 2010, MNRAS, 402, 1536

Shull J. M., 2014, ApJ, 784, 142

Silva M. B., Kooistra R., Zaroubi S., 2016, MNRAS, 462, 1961

Steidel C. C., Erb D. K., Shapley A. E., Pettini M., Reddy N., Bogosavljević M., Rudie G. C., Rakic O., 2010, ApJ, 717, 289

Steidel C. C., Bogosavljević M., Shapley A. E., Kollmeier J. A., Reddy N. A., Erb D. K., Pettini M., 2011, ApJ, 736, 160

Stinson G., Seth A., Katz N., Wadsley J., Governato F., Quinn T., 2006, MNRAS, 373, 1074 
Suresh J., Nelson D., Genel S., Rubin K. H. R., Hernquist L., 2019, MNRAS, 483,4040

Teyssier R., 2002, A\&A, 385, 337

Teyssier R., Pontzen A., Dubois Y., Read J. I., 2013, MNRAS, 429, 3068

Thatte N. A. et al., 2014, in Ramsay S. K., McLean I. S., Takami H., eds, Proc. SPIE Conf. Ser. Vol. 9147, Ground-based and Airborne Instrumentation for Astronomy V. SPIE, Bellingham, p. 914725

Trebitsch M., Verhamme A., Blaizot J., Rosdahl J., 2016, A\&A, 593, A122

Tumlinson J., Peeples M. S., Werk J. K., 2017, ARA\&A, 55, 389

van de Voort F., Springel V., Mandelker N., van den Bosch F. C., Pakmor R., 2019, MNRAS, 482, L85

Verhamme A., Schaerer D., Maselli A., 2006, A\&A, 460, 397

Verhamme A., Dubois Y., Blaizot J., Garel T., Bacon R., Devriendt J., Guiderdoni B., Slyz A., 2012, A\&A, 546, A111

Vogelsberger M. et al., 2014, MNRAS, 444, 1518

Wisotzki L. et al., 2016, A\&A, 587, A98

Wisotzki L. et al., 2018, Nature, 562, 229

Wofford A., Leitherer C., Salzer J., 2013, ApJ, 765, 118

Wold I. G. B., Barger A. J., Cowie L. L., 2014, ApJ, 783, 119

Wold I. G. B., Finkelstein S. L., Barger A. J., Cowie L. L., Rosenwasser B., 2017, ApJ, 848, 108

Zahid H. J., Dima G. I., Kewley L. J., Erb D. K., Davé R., 2012, ApJ, 757, 54

Zieleniewski S., Thatte N., Kendrew S., Houghton R. C. W., Swinbank A. M., Tecza M., Clarke F., Fusco T., 2015, MNRAS, 453, 3754

\section{APPENDIX A: SPECIFICS OF THE HIGH-RESOLUTION SIMULATION}

Fig. A1 shows in green-blue the spatial distribution of new, highresolution gas cells, which were not accessible in Frank et al. (2012). They are mainly associated with ISM-like gas. Fig. A2 shows the spatial distribution of the ISM and the self-shielded gas according to our definition, given in the main text. Fig. A3 gives maps of the typical densities and temperatures in our most massive haloes. Fig. A4 shows the Ly $\alpha$ and O VIII emission maps in the most massive halo at $z=0.3$.

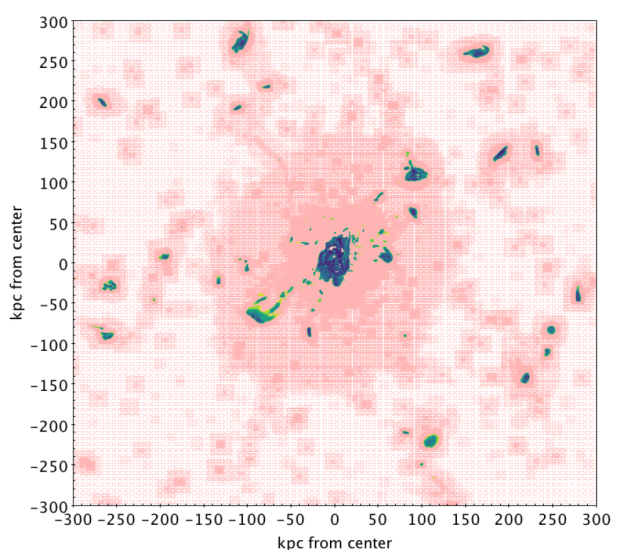

Figure A1. Example for the emergence of discs in our simulation with the green-blue colour indicating the density and temperature combinations that were unreachable with the previous, low-resolution simulation. The redshift of this snapshot is 0.7. The halo shown here is the one we discuss in Section 3 and also for the FIREBall-2 analysis.

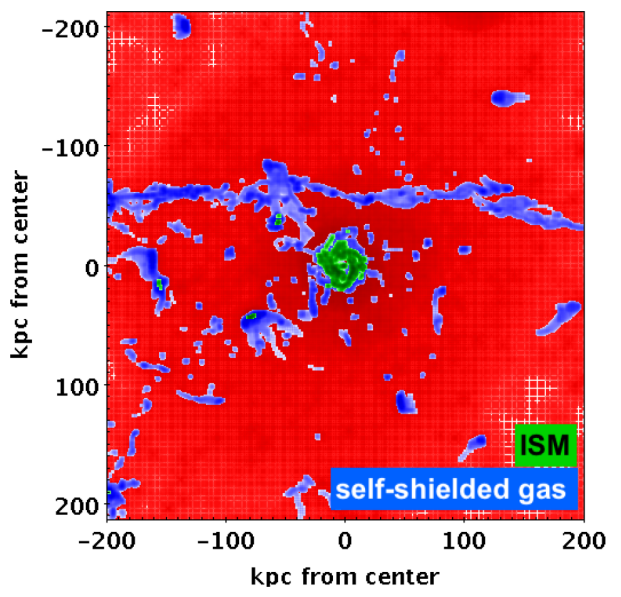

Figure A2. Example of the distribution of gas cells that fall into our definition of self-shielded (blue) and ISM (green). For the chosen cuts on each phase, see Fig. 5. The halo shown here is the one we use for the HARMONI analysis at $z=0.3$.
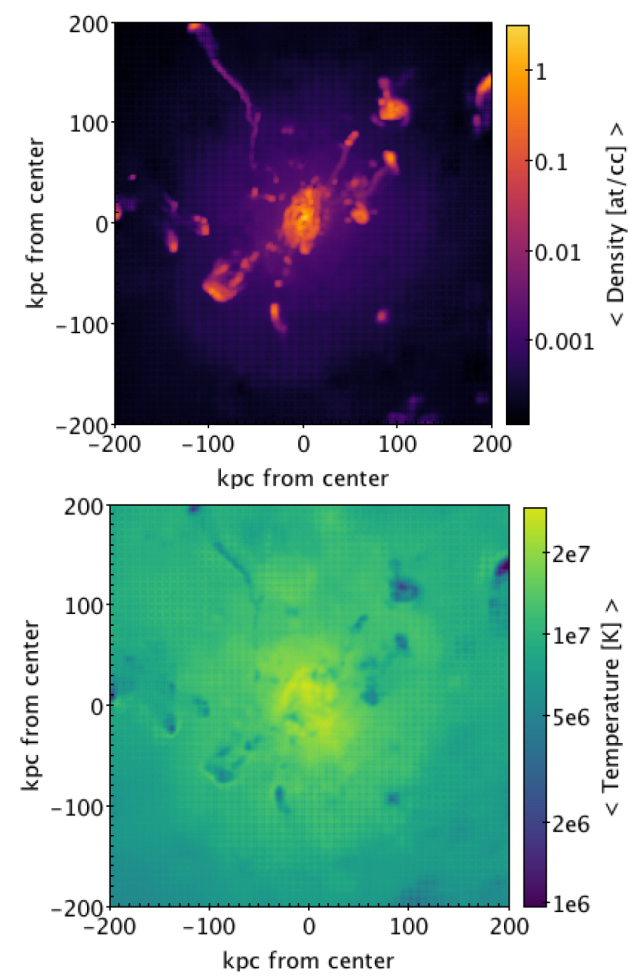

Figure A3. The upper panel shows the total hydrogen density map for the most massive halo at $z=0.7$. The lower panel shows the temperature map of the same halo. The halo shown here is the one we discuss in Section 3 and also for the FIREBall-2 analysis. 


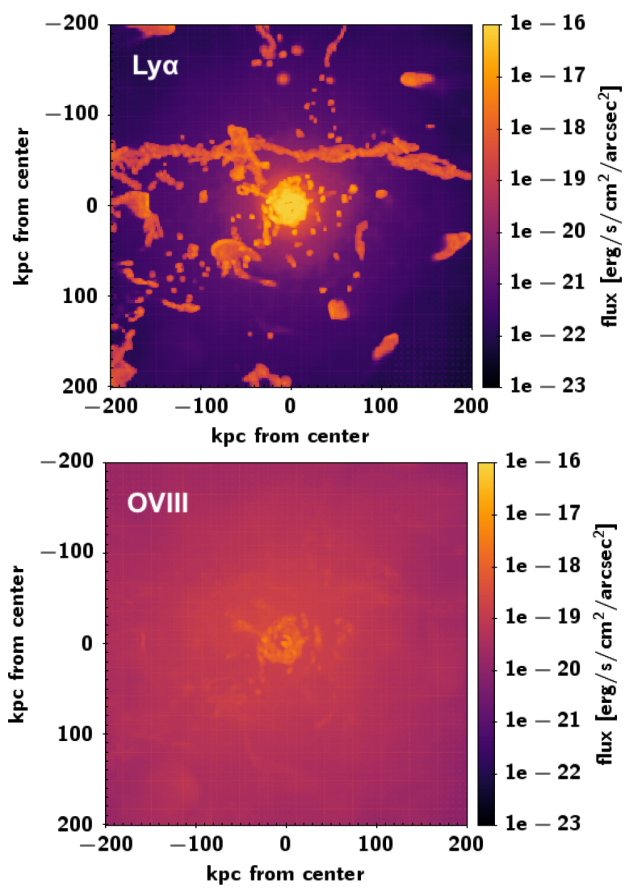

Figure A4. Ly $\alpha$ and O VIII emission maps at $z=0.3$. While the 'cool' hydrogen gas emits strongly from clumps in the halo, the X-ray emission of e.g. $\mathrm{O}$ VIII is more homogeneous throughout the halo. The halo shown here is the one we use for the HARMONI analysis at $z=0.3$.

\section{APPENDIX B: ADDITIONAL INFORMATION ON THE FIREBALL-2 SNR}

Table B1 gives the properties of all the 30 haloes that were used as input to the FIREBall-2 instrument model. Table B2 gives the SNR for a halo with a given input magnitude and the number of objects to be stacked at this magnitude to reach and SNR of 3 .

Table B1. Properties of the haloes that were used as input for the FIREBall IMO. The first column gives the emission line, the second the redshift. Column 3 gives the dark matter mass of the halo and column 4 the stellar mass. The star formation rate is given in column 5 .

\begin{tabular}{lcccc}
\hline Line & Redshift & $\begin{array}{c}M_{\mathrm{DM}} \\
\left(\mathrm{M}_{\odot}\right)\end{array}$ & $\begin{array}{c}M_{\star} \\
\left(\mathrm{M}_{\odot}\right)\end{array}$ & $\begin{array}{c}\mathrm{SFR} \\
\left(\mathrm{M}_{\odot} \mathrm{yr}^{-1}\right)\end{array}$ \\
\hline C IV & 0.3 & $2.83 \mathrm{e}+13$ & $3.37 \mathrm{e}+12$ & 333 \\
C IV & 0.3 & $6.64 \mathrm{e}+12$ & $9.98 \mathrm{e}+11$ & 60 \\
C IV & 0.3 & $3.51 \mathrm{e}+12$ & $5.10 \mathrm{e}+11$ & 37 \\
C IV & 0.3 & $1.64 \mathrm{e}+12$ & $2.34 \mathrm{e}+11$ & 26 \\
C IV & 0.3 & $1.17 \mathrm{e}+12$ & $1.78 \mathrm{e}+11$ & 14
\end{tabular}

Table B1 - continued

\begin{tabular}{|c|c|c|c|c|}
\hline Line & Redshift & $\begin{array}{l}M_{\mathrm{DM}} \\
\left(\mathrm{M}_{\odot}\right)\end{array}$ & $\begin{array}{c}M_{\star} \\
\left(\mathrm{M}_{\odot}\right)\end{array}$ & $\begin{array}{c}\mathrm{SFR} \\
\left(\mathrm{M}_{\odot} \mathrm{yr}^{-1}\right)\end{array}$ \\
\hline C IV & 0.3 & $1.03 \mathrm{e}+12$ & $1.40 \mathrm{e}+11$ & 10 \\
\hline CIV & 0.3 & $6.18 \mathrm{e}+11$ & $7.21 \mathrm{e}+10$ & 7 \\
\hline CIV & 0.3 & $6.04 \mathrm{e}+11$ & $1.82 \mathrm{e}+10$ & 11 \\
\hline CIV & 0.3 & $4.53 \mathrm{e}+11$ & $4.95 \mathrm{e}+10$ & 7 \\
\hline CIV & 0.3 & $4.20 \mathrm{e}+11$ & $4.09 \mathrm{e}+10$ & 4 \\
\hline $\operatorname{Ly} \alpha$ & 0.7 & $1.36 \mathrm{e}+13$ & $1.11 \mathrm{e}+12$ & 246 \\
\hline $\operatorname{Ly} \alpha$ & 0.7 & $5.96 \mathrm{e}+12$ & $7.91 \mathrm{e}+11$ & 169 \\
\hline $\operatorname{Ly} \alpha$ & 0.7 & $3.07 \mathrm{e}+12$ & $4.02 \mathrm{e}+11$ & 82 \\
\hline $\operatorname{Ly} \alpha$ & 0.7 & $2.64 \mathrm{e}+12$ & $3.01 \mathrm{e}+11$ & 96 \\
\hline $\operatorname{Ly} \alpha$ & 0.7 & $1.80 \mathrm{e}+12$ & $1.98 \mathrm{e}+11$ & 63 \\
\hline $\operatorname{Ly} \alpha$ & 0.7 & $1.16 \mathrm{e}+12$ & $1.46 \mathrm{e}+11$ & 24 \\
\hline $\operatorname{Ly} \alpha$ & 0.7 & $1.01 \mathrm{e}+12$ & $1.33 \mathrm{e}+11$ & 39 \\
\hline $\operatorname{Ly} \alpha$ & 0.7 & $9.98 \mathrm{e}+11$ & $5.65 \mathrm{e}+10$ & 40 \\
\hline $\operatorname{Ly} \alpha$ & 0.7 & $9.04 \mathrm{e}+11$ & $9.79 \mathrm{e}+10$ & 32 \\
\hline $\operatorname{Ly} \alpha$ & 0.7 & $8.40 \mathrm{e}+11$ & $7.55 \mathrm{e}+10$ & 18 \\
\hline O VI & 1.0 & $9.61 \mathrm{e}+12$ & $8.52 \mathrm{e}+11$ & 622 \\
\hline O VI & 1.0 & $5.85 e+12$ & $5.50 \mathrm{e}+11$ & 333 \\
\hline O VI & 1.0 & $2.86 \mathrm{e}+12$ & $2.76 \mathrm{e}+11$ & 141 \\
\hline O VI & 1.0 & $2.71 \mathrm{e}+12$ & $3.17 \mathrm{e}+11$ & 167 \\
\hline OVI & 1.0 & $2.39 \mathrm{e}+12$ & $2.28 \mathrm{e}+11$ & 99 \\
\hline $\mathrm{OVI}$ & 1.0 & $1.49 \mathrm{e}+12$ & $1.44 \mathrm{e}+11$ & 92 \\
\hline O VI & 1.0 & $1.41 \mathrm{e}+12$ & $1.66 \mathrm{e}+11$ & 88 \\
\hline O VI & 1.0 & $1.28 \mathrm{e}+12$ & $9.45 \mathrm{e}+10$ & 69 \\
\hline O VI & 1.0 & $1.03 \mathrm{e}+12$ & $9.16 \mathrm{e}+10$ & 53 \\
\hline O VI & 1.0 & $9.76 \mathrm{e}+11$ & $9.01 \mathrm{e}+10$ & 45 \\
\hline
\end{tabular}

Table B2. SNR results for $\mathrm{Ly} \alpha$ galaxies with different magnitudes. The first column gives the magnitude, the second the calculated SNR according to equations (15) and (16), corresponding to the pessimistic and the optimistic case. The third column gives the number of targets that need to be stacked at the given magnitude in order to reach an SNR of 3 for the two extreme cases.

\begin{tabular}{lcc}
\hline $\begin{array}{l}\text { Input NUV } \\
\text { AB magnitude }\end{array}$ & $\begin{array}{c}\text { Predicted SNR } \\
\text { per resolution element }\end{array}$ & $\begin{array}{c}\text { Stack } \\
(\mathrm{SNR}=3)\end{array}$ \\
\hline 18 & $3.15-3.97$ & $1-1$ \\
19 & $1.26-2.51$ & $2-6$ \\
20 & $0.50-1.58$ & $4-36$ \\
21 & $0.20-0.99$ & $9-227$ \\
22 & $0.079-0.63$ & $23-1433$ \\
23 & $0.032-0.40$ & $56-9043$ \\
24 & $0.013-0.25$ & $144-57056$ \\
25 & $0.0050-0.16$ & $352-360000$ \\
\hline
\end{tabular}

This paper has been typeset from a $\mathrm{T}_{\mathrm{E}} \mathrm{X} / \mathrm{L} \mathrm{T} \mathrm{E} \mathrm{X}$ file prepared by the author. 